Florida International University FIU Digital Commons

FIU Electronic Theses and Dissertations

University Graduate School

$11-15-2017$

\title{
Using Unmanned Aerial Vehicles for Wireless Localization in Search and Rescue
}

Virgilio Acuna

Florida International University, heartjc@aol.com

DOI: 10.25148 /etd.FIDC006591

Follow this and additional works at: https://digitalcommons.fiu.edu/etd

Part of the Electrical and Computer Engineering Commons

\section{Recommended Citation}

Acuna, Virgilio, "Using Unmanned Aerial Vehicles for Wireless Localization in Search and Rescue" (2017). FIU Electronic Theses and Dissertations. 3646.

https://digitalcommons.fiu.edu/etd/3646

This work is brought to you for free and open access by the University Graduate School at FIU Digital Commons. It has been accepted for inclusion in FIU Electronic Theses and Dissertations by an authorized administrator of FIU Digital Commons. For more information, please contact dcc@fiu.edu. 


\title{
FLORIDA INTERNATIONAL UNIVERSITY
}

Miami, Florida

\section{USING UNMANNED AERIAL VEHICLES FOR WIRELESS LOCALIZATION IN SEARCH AND RESCUE MISSIONS}

A thesis submitted in partial fulfillment of the requirements for the degree of MASTER OF SCIENCE

in

\section{ELECTRICAL ENGINEERING}

by

\author{
Virgilio Acuna
}


To: Dean John L. Volakis

College of Engineering and Computing

This thesis, written by Virgilio Acuna, and entitled Using Unmanned Aerial Vehicles for Wireless Localization in Search and Rescue Missions, having been approved in respect to style and intellectual content, is referred to you for judgment.

We have read this thesis and recommend that it be approved.

Leonardo Bobadilla

Selcuk Uluagac

Ismail Guvenc, Major Professor

Date of Defense: November 15, 2017

The thesis of Virgilio Acuna is approved.

Dean John L. Volakis

College of Engineering and Computing

Andrés G. Gil

Vice President for Research and Economic Development and Dean of the University Graduate School

Florida International University, 2018 
ABSTRACT OF THE THESIS

USING UNMANNED AERIAL VEHICLES FOR WIRELESS LOCALIZATION IN SEARCH AND RESCUE MISSIONS

by

Virgilio Acuna

Florida International University, 2018

Miami, Florida

Professor Ismail Guvenc, Major Professor

This thesis presents how unmanned aerial vehicles (UAVs) can successfully assist in search and rescue (SAR) operations using wireless localization. The zone-grid to partition to capture/detect WiFi probe requests follows the concepts found in Search Theory Method. The UAV has attached a sensor, e.g., WiFi sniffer, to capture/detect the WiFi probes from victims or lost people's smartphones. Applying the Random-Forest based machine learning algorithm, an estimation of the user's location is determined with a $81.8 \%$ accuracy.

UAV technology has shown limitations in the navigational performance and limited flight time. Procedures to optimize these limitations are presented. Additionally, how the UAV is maneuvered during flight is analyzed, considering different SAR flight patterns and Li-Po battery consumption rates of the UAV. Results show that controlling the UAV by remote-controll detected the most probes, but it is less power efficient compared to control it autonomously. 


\section{TABLE OF CONTENTS}

CHAPTER

PAGE

I INTRODUCTION .1

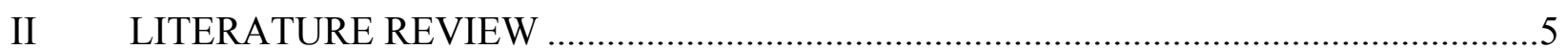

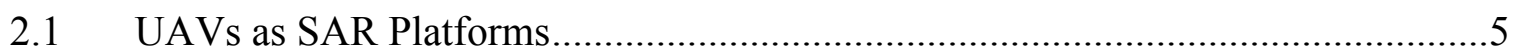

$2.2 \quad$ Wireless Localization......................................................................................

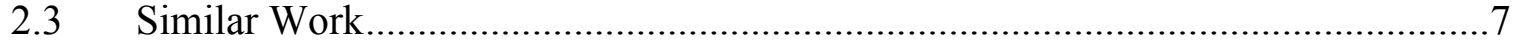

III LOCALIZATION OF WIFI DEVICES USING PROBE REQUESTS CAPTURED AT

UNMANNED AERIAL VEHICLE …………………...........................................

3.1 Environmental Setup for the Experimentation ....................................................

3.1.1 Wi-Fi Pineapple ...........................................................................

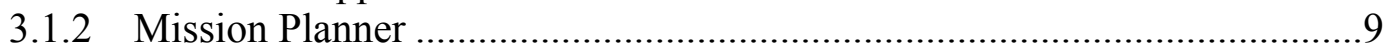

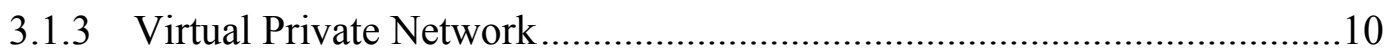

3.2 Capturing Data with WIFI Pineapple …………………………........................12

3.2.1 UAV Deployment to Capture Wi-Fi Probe Requests .................................12

3.2.2 UAV Flight Trajectory to Capture Wi-Fi Probe Requests............................12

3.2.3 Using Wi-Fi Pineapple to Capture Wi-Fi Probe Requests...........................14

3.2.4 Wi-Fi Probe Requests Traffic Capturing using Wireshark..........................14

$3.3 \quad$ Random Forest Algorithm ………………………............................................15

$3.4 \quad$ Numerical and Experimental Results...............................................................17

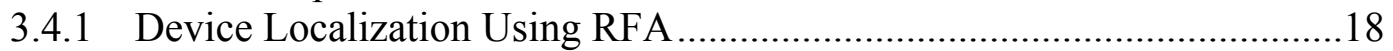

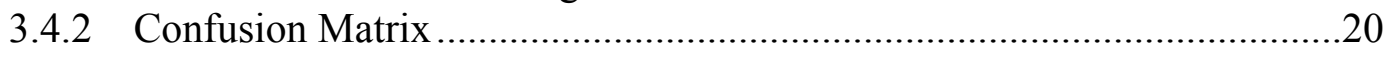

3.4.3 Results Analysis .............................................................................21

IV UAV PROCEDURRES TO EXTEND FLIGHT TIME FOR SEARCH AND RESCUE

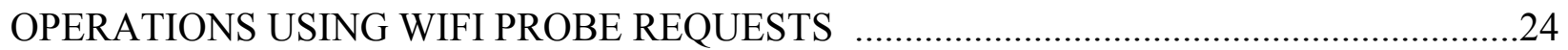

4.1 Environment of Experimental Design ...........................................................24

4.1.1 Sensor to Detect Wi-Fi Probes...................................................................24

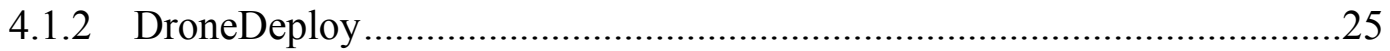

4.1.3 FAA Rules and Regulations for UAVs..............................................25

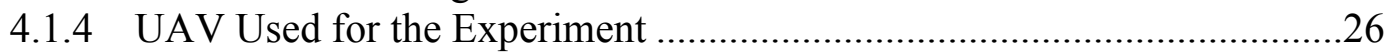

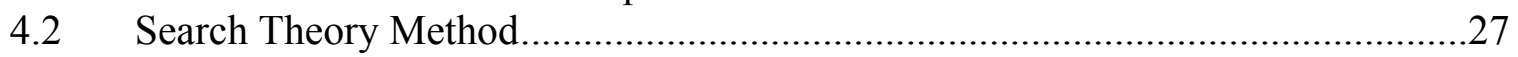

4.2.1 Probability of Containment ..................................................................2

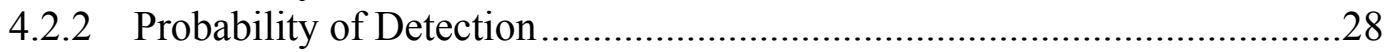

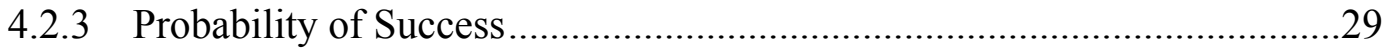

4.3 Ways to Maximize UAV Flight Time …………...............................................30

4.3.1 Optimization of Propellers ........................................................................

4.3.2 LiPo Battery Basics.................................................................................... 32

4.4 SAR Flight Patterns Follow by UAV Affect Optimization of UAV Flight Times

.

4.4.1 Parallel Track Search Pattern (PTSP) …………........................................39

4.4.2 Creeping Line Search Pattern (CLSP) ......................................................41 
4.4.3 Sector Search Pattern (SSP)

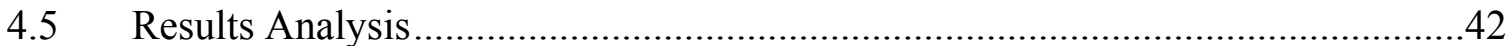

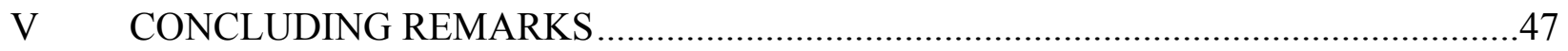

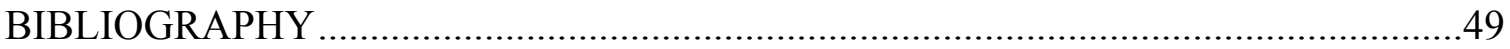




\section{LIST OF TABLES}

TABLE

PAGE

1. Example for Captured WiFi probe request packets for a duration of 6 seconds ..............15

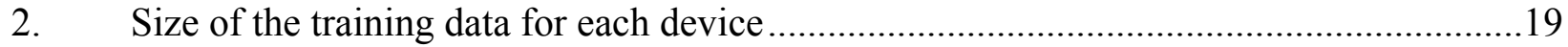

3. Confusion matrix constructed using captured data................................................... 19

4. SAR Flight data obtained from DroneDeploy ........................................................43

5. Results with an autonomous UAV over different SAR flight patterns...........................44

6. Results with a remote-controlled UAV over different SAR flight patterns....................45 


\section{LIST OF FIGURES}

FIGURE

PAGE

1. (a) Experimental setup with WiFi Pineapple mounted on a drone, (b) Model used to access packets from WiFi Pineapple remotely to localize a WiFi device

2. UAV flight plan used for WiFi localization ............................................................ 11

3. Unique devices identified from different manufacturers based on the WiFi probe requests captured at the UAV....

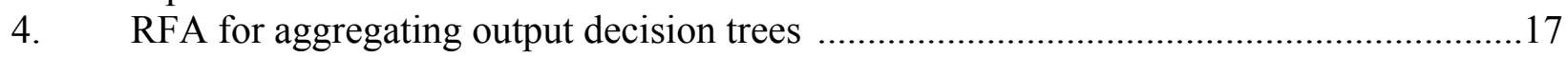

5. Classification of WiFi device into different zones using the longitude, latitude, altitude,

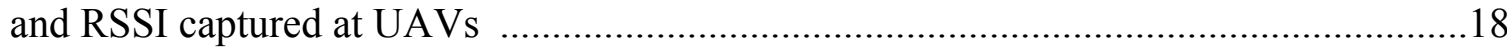

6. Accelerometer sensors' threshold to resist vibration [56].........................................32

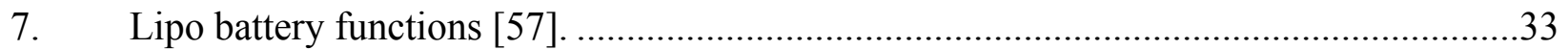

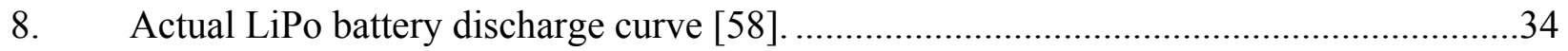

9. Relationship between flight time and LiPo capacity [59]........................................38

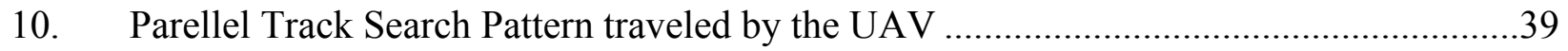

11. Creeping Line Search Pattern traveled by the UAV ...............................................40

12. Sector Search Pattern traveled by the UAV ................................................................42 


\title{
CHAPTER I
}

\author{
Introduction
}

Search and Rescue Emergency scenarios demand a rapid response from first responders to gather information about the localization of victims and reach them promptly. A scenario, for example, can be people who are left trapped inside a building after it collapsed, including unconscious victims, without the availability of a wireless connection to call for assistance and disclose their location to the search and rescue personnel $[1,2]$. Search and Rescue (SAR) scenarios involved natural and human-made disasters that can cause damages to the existing cellular infrastructure [3] and situations in which the victims may have stepped outside the cellular coverage area, as in the case of lost hikers in national parks [4]. What is the most effective and systematic way of searching an area of distress to localize lost people or affected victims? According to the national SAR manual, which is the standard reference document adopted by all the SAR communities, the grid (zone) searching pattern is defined as the most effective search pattern to localize either lost people or distressful victims during SAR Emergency scenarios.

SAR operations involve temporal and spatial components that must be considered when planning and managing a mission. Detecting the location of the victims in the least possible amount of time is critical since any delays will likely reduce the chances of survival [27]. Obtaining a rapid overview of the situation becomes crucial, and the sky supplies a mobile vantage position to deploy assistance at the right time and location. Gathering the maximum data over the affected area or designated search area provides a wide-area situational awareness for distributing the scarce resources of time and rescue team/equipment. With many onboard sensors (e.g., thermal and optical cameras, LIDAR, remote sensing devices, and GPS), an Unmanned Aerial Vehicle (UAV) can capture data during SAR operations and transfer it to a ground base station in real time [28]. 
Recent advances in hardware and software technology have allowed UAVs to perform a broad set of tasks and enable their use in many different civilian applications, including SAR, mapping, and surveillance of a region of interest. UAVs have shown to be a promising and powerful technology to conduct SAR operations safer, faster, and more efficiently due to their agility, portability, and aerial advantages. Furthermore, UAVs reduce the likelihood of exposing SAR members to unnecessary danger during SAR operations and provide access to remote or difficult-to-reach locations deemed dangerous for the SAR crew or those that take a long time to reach. The introduction of UAVs will make disaster relief operations faster and safer [29].

As UAVs are becoming more ubiquitous, the SAR communities have capitalized on their use to assist and supplement in a search procedure or disaster response and relief [30, 31,32]. In an UAV-based search and rescue operation, a good line of sight and the discretization of the search area render a high probability of localizing a victim in a given zone [9]. Hence, UAVs can employ different search strategies to localize victims in a given designated search area. An image processing technique to locate victims is used by the UAVs in $[10,11]$.

However, given the limited visibility and cluttering conditions observed during the emergency, it might not be possible to accurately detect and track individuals using wireless cameras or real-time video, especially from longer distances. As an alternative to the camera vision-based localization of victims, Wi-Fi signals from victims' wireless devices may also be used by UAVs for SAR operations $[12,13]$. Attached to the UAV is the Tetra Wi-Fi Pineapple sensor to detect Wi-Fi probe requests, which are a ubiquitous feature used in smartphones, enabled by default and rarely ever disabled by the user. Smartphones use probe request transmissions to scan and to find Wi-Fi access points (APs) $[47,48,49]$. The smartphones send out probe requests to connect to previous APs or discover new APs available in the vicinity. Probe request transmissions, i.e., beacons, take place every few seconds, and the smartphone can be detected if 
it is within signal range, even if the user does not connect to the APs. By deploying a packet analyzer tool to sniff Wi-Fi, Tetra Wi-Fi Pineapple [50] detects the probe requests transmitted by smartphones and can potentially reduce the area of interest to search during SAR operations. Hence, a machine learning technique is used to localize Wi-Fi devices by capturing/detecting probes using a UAV.

The use of a UAV as a tool for SAR operations contains limitations. The flight time of a UAV is limited. The flight performance of the UAV can be affected by the addition of weight to its overall payload mass. For example, the UAV stability performance controlled by the embedded navigational sensors found in the flight controller can be reduced, the already limited flight time can be further lessened, and the climb rate reduced, especially during autonomous flight in altitude hold and hovering flight mode. Not only the navigational performance of the UAV is reduced, but also the power consumption of the UAV works harder by drawing more energy from the battery. The search trajectory set by the SAR crew for the UAV to fly also has an adverse impact on the flight time, the power consumption of the UAV, and the number of probes detected/collected.

In this thesis, a machine learning algorithm is used to predict the correct occupancy zone of Wi-Fi devices from a geographical zone grid division. The chosen algorithm for this application depends on the size and quality of the data and the extracted parameters from the sensor used. Then, the algorithm classifies the collected observations and predicts an outcome with high accuracy.

The thesis also addresses the limitations posed by the UAV technology. It presents in-depth procedures to maximize the flight time of the UAV, improves its flight performance, and how the sensory operations are affected in detecting probes by the search flight patterns flown by the UAV.

The thesis is organized in the following order: 
Chapter 2 discusses a literature review about the main issues on Wi-Fi localization and UAV technology.

Chapter 3 concentrates on the localization of Wi-Fi devices using probe requests captured by Unmanned Aerial Vehicles.

Chapter 4 presents procedures to extend the flight time of Unmanned Aerial Vehicles and flight patterns detecting the most probes.

Chapter 5 draws conclusions on Wi-Fi localization captured by Unmanned Aerial Vehicles, processes to extend their flight time, the effective flight pattern to detect probes, and future studies. 


\title{
CHAPTER II
}

\author{
Literature Review
}

\subsection{UAVs as SAR Platforms}

UAV's platforms have rapidly begun to be adopted for search and rescue operations $[5,6,8]$. The SAR teams have realized the lifesaving capabilities of UAVs due to their easy deployability and airborne characteristics that render a good line of sight with the ground entities. Once they are deployed to conduct a flight mission, UAVs can monitor the designated search areas using various sensors mounted onto their frames and connected to the flight controller. UAVs can be used as aerial platforms to collect images to monitor a disaster area $[33,34]$; to rescue natural disaster victims and transport medical samples and supplies [35]; to carry a payload of different visual sensors and digital image stabilization circuit to stabilize thermal and RGB video to support first responders in disaster scenarios [36, 37]; to generate fast and actionable 3D maps modeling a disaster scene for emergency response [38]; to provide orthomosaics images of the data captured during a SAR flight mission to assist responding agencies [39]; and to use RGB-Depth sensors to build 3D point cloud models of disaster scenes and import them into a state-of-the-art game engine for first responders to interact with the simulated environment [40].

Furthermore, an essential role of UAVs in SAR operations has been a combination of aerial photography, including associated pattern recognition and image processing algorithms [41] and remote image sensing [42, 43, 44]. Thermal sensitivity and zoom capabilities, enhanced IR sensors, and a higher heat detection combined with pan tilt zoom capabilities are some of the advanced features provided by thermal imaging cameras that can be mounted onto the UAV for SAR operations. With the designed image recognition algorithm captured by the UAV, the technology has shown levels of success by detecting the search objects or victims. Hence, UAVs 
are capable and adaptable to be used, as aerial platforms to collect disaster scenes data over designated search areas that used to be carried out by conventional airborne platforms.

\subsection{Wireless Localization}

As an alternative to the camera vision-based localization of victims, Wi-Fi signals from victims' wireless devices may also be used by UAVs for search [12, 13, 14, 15, 16, 17].

This search strategy can help in delivering food and medical packages using UAVs [12], analyzing pedestrian/vehicular traffic pattern during evacuation [18], and identifying the indoor location of people stuck in a fire building [19]. Therefore, using a passive sniffer, such as a Wi-Fi Pineapple [21], it is possible to capture and decode ambient Wi-Fi probe requests broadcasted by Wi-Fi devices, such as smartphones and tablets [20]. To find a Wi-Fi device's location, we extract the received signal strength indicator (RSSI), unique MAC address of the Wi-Fi devices from the WiFi probe requests, and record them at different locations of a UAV (captured using GPS).

While triangulation based techniques can be used to estimate the location of the Wi-Fi device from an estimated distance at different locations [19], for localization in 3D scenarios, RSSI no longer depends monotonically on the distance and can show significant variations, as a function of the UAV elevation [See Fig. 4].

\subsection{Similar Work}

In [51], a UAV is used to reliably detect Wi-Fi-enabled mobile phones from relatively long ranges, giving the sensor's range capability. From the air, the UAV detects the probes, and on the ground, the mobile phones have a custom application installed to extend the battery life and to continue sending Wi-Fi frames, even while the phones' display is turned off. As long as the phones remain on, they will continue to broadcast Wi-Fi probe requests scanning for any active Wi-Fi access point in the vicinity. In [61], a UAV captures Wi-Fi probes in a zone-grid area flying at different 
heights. Smartphones are placed in each partition of the zone-grid area, and the UAV captures the Wi-Fi probe requests to determine the localization of each device.

In this thesis, a different approach is taken in that the UAV is kept in the air the longest possible amount of time by implementing the procedures outlined to extend the flying time of the UAV. Keeping the UAV longer in the air is crucial for the continuous capture/detection of Wi-Fi probes to collect more probes and increase the probability of their localization. The more probes, the better the prediction model, e.g., Random Forest Algorithm, to associate a Wi-Fi device accurately with the correct zone that provides a coarse location information about the Wi-Fi devices.

However, UAVs have a downside of additional power consumption to the addition of sensors and flight maneuvers while conducting SAR operations. Additional weight to the payload mass of the UAV takes energy away from the battery and affects the duration of the overall flight time. Similar works $[45,46]$ have also addressed the effect of weight on the flight duration of a UAV and have simulated the energy consumption of the UAV battery on various flight scenarios. The analysis in this thesis uses a different approach by flying in real time a UAV autonomously and manually to show how flight patterns affect the battery performance. The concepts of propellers' calibration and functions of Lithium Polymer (LiPo) batteries are analyzed. These concepts show either a positive or negative effect on the navigational performance, which is crucial to have during SAR missions, and either reducing or extending the flight time of the UAV, which is a significant constraint to the limited time the aircraft has in the air. These concepts have had limited analysis in real time in the literature. Furthermore, this work differs in that different actual search patterns are flown, and how each pattern is more efficient at detecting probes. The flight pattern and search area are determined by understanding the type of terrain and search object, as outlined by Search Theory Method. 


\section{CHAPTER III}

Localization of Wi-Fi Devices Using Probe Requests Captured at Unmanned Aerial Vehicles

\subsection{Environmental Setup for the Experimentation}

\subsubsection{Wi-Fi Pineapple}

The Wi-Fi Pineapple Mark V is a wireless network auditing tool [21], which is accessible remotely via a Wi-Fi interface. It is power up using a rechargeable battery connected to its DC port. The rechargeable battery is light in weight, making it portable and ideal to use in the experiment. The Wi-Fi Pineapple has dual Atheros AR9331 system on a chip with integrated radios, including a $400 \mathrm{MHz}$ MIPS processor, a 16 MB ROM, and a 64 MB RAM. The Wi-Fi Pineapple is accessible through a web interface or an inbuilt Unix machine called BusyBox [22]. In this experiment, the Wi-Fi Pineapple equipment [23] is mounted onto a Tarot 650 UAV as shown in Fig. 1.

\subsubsection{Mission Planner}

Mission planner is a ground control station that can be used to remotely control a plane, copter, UAV, or rover [24]. It is used as a configuration utility or a dynamic control supplement for autonomous vehicles. Using mission planner, we can analyze the telemetry logs obtained from the UAV and stored on the SD card. The telemetry log includes the communication messages between the ground station and the UAV. These messages are known, as the MAVlink command messages, and they provide information on the UAV flight path, dynamics, and status. The real-time GPS coordinates of the UAV, needed for this experiment are extracted from the telemetry logs. The GPS coordinates are expressed 


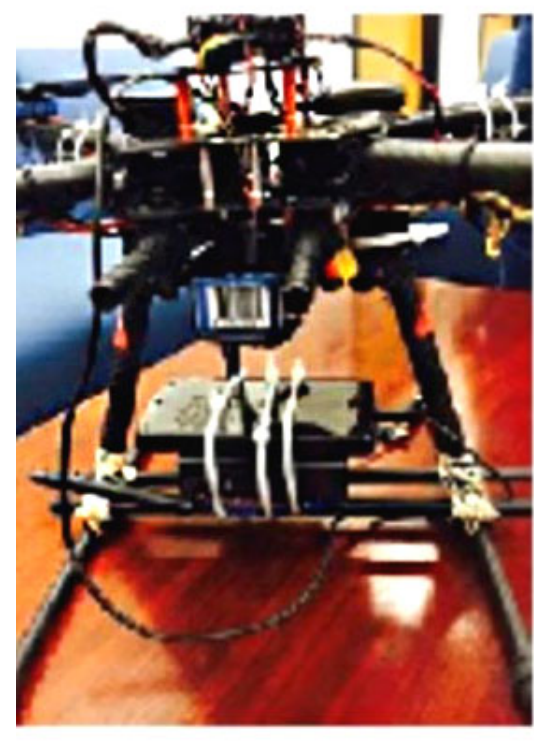

(a)

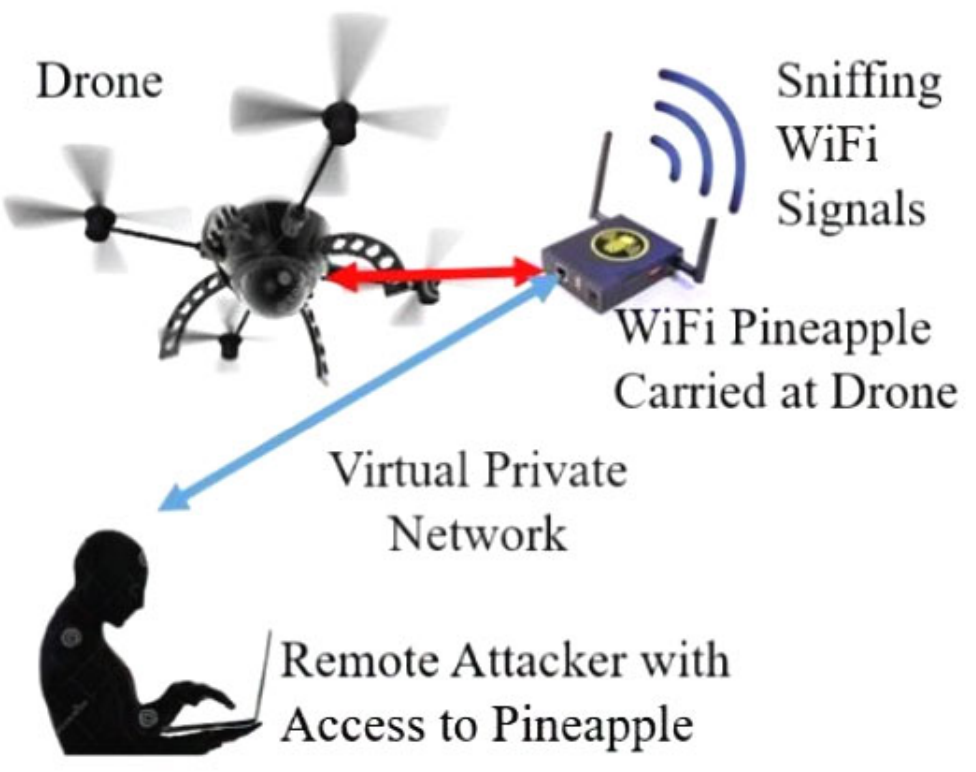

(b)

Fig. 1. (a) Experimental setup with Wi-Fi Pineapple mounted on a drone, and (b) Model used to access packets from Wi-Fi Pineapple remotely to localize a Wi-Fi device.

as a combination of the latitude, longitude, and altitude. The altitude information enables a 3D localization of the Wi-Fi devices on the ground.

\subsubsection{Virtual Private Network}

The experimental setup of the Wi-Fi Pineapple mounted onto the UAV is shown in Fig. 1(a). We can access the Wi-Fi (a) Google Earth image of the path traveled by the UAV and (b) Processed path in the coordinate system, as illustrated in Fig. 2. However, the UAV, having the Wi-Fi Pineapple mounted onto the bottom of the frame, will be flying at different heights and remote locations. Therefore, a smartphone is mounted onto the UAV 


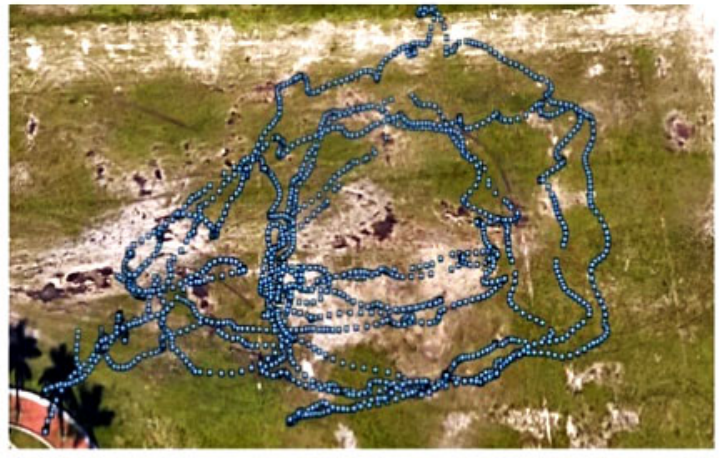

(a)

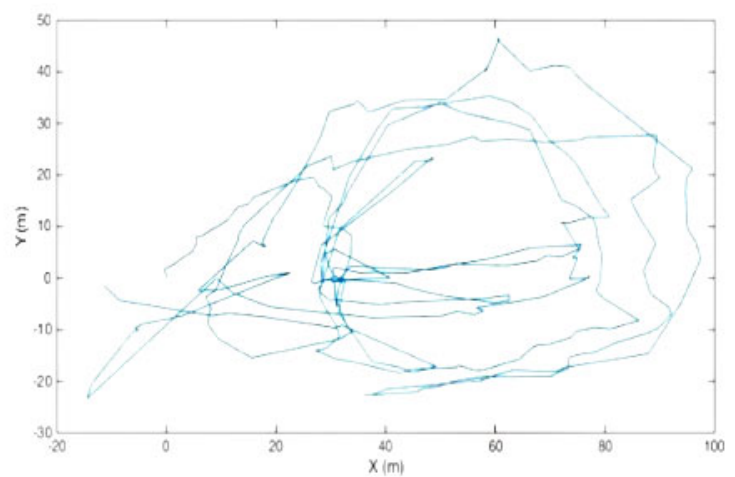

(b)

Fig. 2. UAV flight plan used for Wi-Fi localization.

to provide Internet connection to the Wi-Fi Pineapple. Alternatively, a USB modem can also be used, instead of a smartphone, to reduce the payload on the UAV. Through the Internet connection, we establish a virtual private network (VPN) connection between the Wi-Fi Pineapple and the user terminal, as illustrated in Fig. 1(b). The experiment used an open-source software application called OpenVPN that implements VPN. OpenVPN uses the OpenSSL library to provide encryption of the data and the control channels. The public safety data available captured by the Wi-Fi Pineapple and the UAV during SAR operations can be critical and sensitive. Therefore, a VPN connection ensures a secure communication between the UAV and the emergency first responders' terminal. The VPN connection presents an ability to safeguard the public safety data from any possible cybersecurity threat.

\subsection{Capturing Data with WI-FI Pineapple}

\subsubsection{UAV Deployment to Capture Wi-Fi Probe Requests}

The UAVs fall into two broad categories, as either rotary wings or fixed wings. Given the application of this experiment, a Tarot 650 rotary-wing UAV is utilized. The Tarot 650 quadcopter, since it has 4 propellers, is equipped with a Pixhawk autopilot suitable for a rotary wing UAV, 
having autonomous navigation, a power source, and telemetry. It can communicate to a ground base station and capable of carrying a payload of $6 \mathrm{lbs}$. The blueprint for a random flight plan of the UAV can be obtained from the mission planner ground control station. Furthermore, we also use the mission planner to create the KML and the GPX files of the UAV flight plan. These files record the GPS flight data from the very beginning the UAV is connected to the mission planner via telemetry and generate the map annotation to open with Google Earth, as shown in Fig. 2a, and view the flight plan in the coordinate system, as shown in Fig. $2 \mathrm{~b}$. The telemetry radios are used to connect wirelessly to the UAV and to communicate with the ground control station from the air, using the MAVLink protocol. During SAR missions, this interactive communication link allows the reception of real-time data from the UAV. The telemetry radios are connected to the user's laptop or tablet using a USB cable, and the laptop serves as the ground control station. The approximate range of telemetry radios is about one mile and on a periodic basis provide the UAV status.

\subsubsection{UAV Flight Trajectory to Capture Wi-Fi Probe Requests}

The Federal Aviation Administration (FAA) has created the National Airspace System (NAS) to establish standard operating procedures and regulations for UAV operations in 


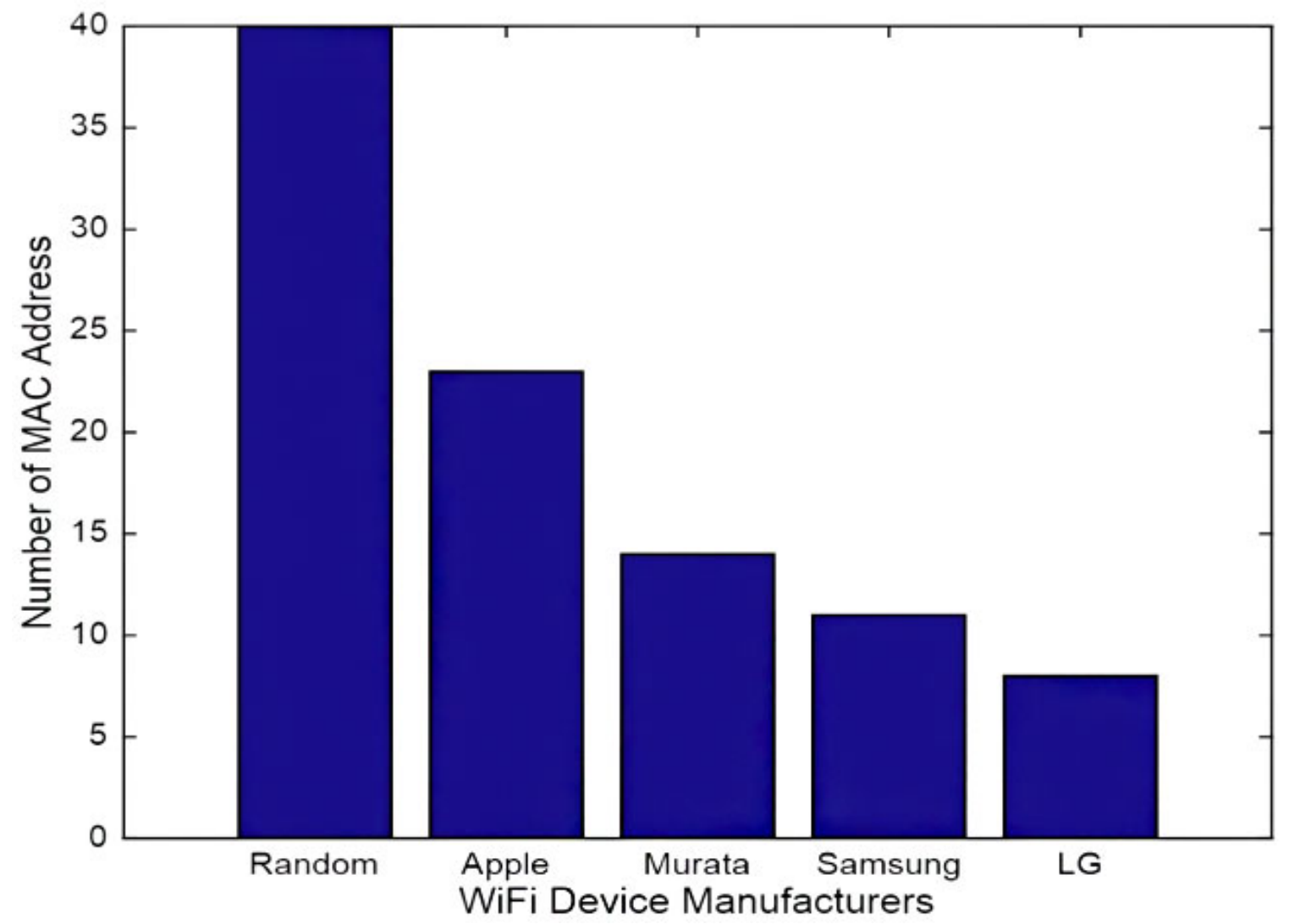

Fig. 3. Unique devices identified from different manufacturers based on the Wi-Fi probe requests captured at the UAV.

the civilian airspace safely and efficiently. The FAA newer provisions to Part 107 integrates the UAVs into the NAS . The FAA rules prohibit any operators to fly a UAV beyond 400 feet and beyond the operator's line of sight. Moreover, the flights must not operate within five miles of any major airports and two miles from any heliports. In our experiment, the UAV operates following all the FAA regulations. The test cellphones are positioned in the field in the form of a grid. All the test cellphones are separated from each other a distance of 24 feet, as shown in Fig. 5. In our experiment, the UAV follows a flight pattern used for mapping missions. In this flight plan, the UAV flies in a circular pattern at different altitudes over the geographical area of interest to capture Wi-Fi probe requests. 


\subsubsection{Using Wi-Fi Pineapple to Capture Wi-Fi Probe Requests}

In our experimentation, we automate the scripts to control Wi-Fi Pineapple through the customizable boot modes. Using the Wi-Fi Pineapple web interface, we specify the script to run on boot up, and on subsequent bootup, the Wi-Fi Pineapple executes the commands listed in the script. Once the Internet connection is established, the date and time in the Wi-Fi Pineapple are updated using an NTP server. Upon boot up, the Wi-Fi Pineapple starts capturing Wi-Fi probe requests in the $2.4 \mathrm{GHz}$ band. The Wi-Fi Pineapple also captures the Wi-Fi packets originating from the nonexperimental Wi-Fi devices that are within the range of the Wi-Fi Pineapple. The data obtained by the Wi-Fi Pineapple is time stamped and includes the MAC address, the RSSI, and the manufacturer's name of the Wi-Fi enabled devices. Based on the Wi-Fi probe requests captured, Fig. 3 illustrates the different type of devices detected during the flight trajectory of the UAV.

\subsubsection{Wi-Fi Probe Requests Traffic Capturing using Wireshark}

To obtain the live Wi-Fi probe requests captured by the tcpdump analyzer, we SSH into the Wi-Fi Pineapple over the VPN tunnel. The captured packets are dumped into the console of the ground control station, and then, they are further analyzed using Wireshark packet analyzer. These packets

contain SSIDs, RSSIs, timestamps, MAC addresses, and packets count. However, we only retain Wi-Fi probe request packets relevant to our experiment, as shown inTable I. These are the Wi-Fi devices we are interested in localizing. 


\begin{tabular}{|c|c|c|c|c|c|c|c|}
\hline $\begin{array}{c}\text { Packet } \\
\text { number }\end{array}$ & $\begin{array}{c}\text { Time } \\
\text { stamp }\end{array}$ & $\begin{array}{c}\text { RSSI } \\
(\mathbf{d B m})\end{array}$ & $\begin{array}{c}\text { MAC } \\
\text { Address }\end{array}$ & $\begin{array}{c}\text { Device } \\
\text { number }\end{array}$ & Latitude & Longitude & $\begin{array}{c}\text { Elevation } \\
\text { (ft.) }\end{array}$ \\
\hline 857 & $20: 02: 55$ & -65 & $10: c 3: 7 b$ & 3 & 25.7491 & -80.37400 & 35.10499 \\
\hline 860 & $20: 02: 55$ & -63 & $10: c 3: 7 b$ & 3 & 25.7491 & -80.37400 & 35.10499 \\
\hline 863 & $20: 02: 58$ & -75 & AsustekC & 2 & 25.7491 & -80.37403 & 36.28609 \\
\hline 866 & $20: 03: 00$ & -67 & SamsungE & 4 & 25.7491 & -80.37400 & 37.86089 \\
\hline 870 & $20: 03: 01$ & -56 & SamsungE & 4 & 25.7491 & -80.37410 & 39.63255 \\
\hline 879 & $20: 03: 01$ & -67 & MurataMa & 5 & 25.7491 & -80.37412 & 39.30446 \\
\hline
\end{tabular}

TABLE I. Example for Captured WiFi probe request packets for a duration of 6 seconds.

The GPS coordinates are obtained by synching the time of the Wi-Fi Pineapple and the flight controller internal clock. The timestamp of each captured Wi-Fi probe request is then mapped to its corresponding GPS coordinate.

\subsection{Random Forest Algorithm}

In this thesis, the random forest machine learning algorithm [25] is used to localize the Wi-Fi devices into the predefined zones, based on the observation of RSSIs from the Wi-Fi devices. The Random Forest Algorithm (RFA) produces a high accuracy classifier with a low error rate, which is the main motivation for choosing this technique. Furthermore, it can efficiently run on large databases, handle thousands of input variables without variable deletion, provide estimates of what variables are important in the classification, provide an effective methodology for estimating missing data, and build the models quickly where large samples are employed. Therefore, in this experimentation, we analyze a larger number of observations to provide an accurate localization of the Wi-Fi devices using the RFA. 
The RFA is a combination of machine learning methods, using an ensemble of decision trees in which each node is split, using the best among a subset of predictors. The main goal is to reduce the variance of the predictor while the bias remains the same. A randomness is injected into the forest by applying bagging (bootstrap aggregation), where the labeled training data is randomly sampled [26]. The variance reduction of bagging the tree-growing process is improved by using a random selection of the input variables. The intuition behind this approach is to build a strong classifier utilizing a combination of weak classifiers [26].

As illustrated in Fig. 4, the RFA employs decision trees that place data into a categorical classification and uses an aggregating approach. Rather than just using and growing one tree, the random forest utilizes the aggregating output, considering many shallow trees. The reason behind this is that aggregating the errors from all the shallow trees provide a better accurate prediction to the overall classification objective. Each decision tree is a weak learner. However, the RFA is a combination of all weak trees to create a strong learner. The RFA has terminal nodes lying at the bottom of the decision tree. The algorithm starts at the top and then traverses down by choosing a small sample of data at random, creating smaller subsets, starting at Tree 1, Tree 2, and until reaching a total number of Tree $\mathrm{B}$ to create a Random Forest. The objective is to combine all the smaller subsets, which are individual decision trees, to model a single forecast prediction. 


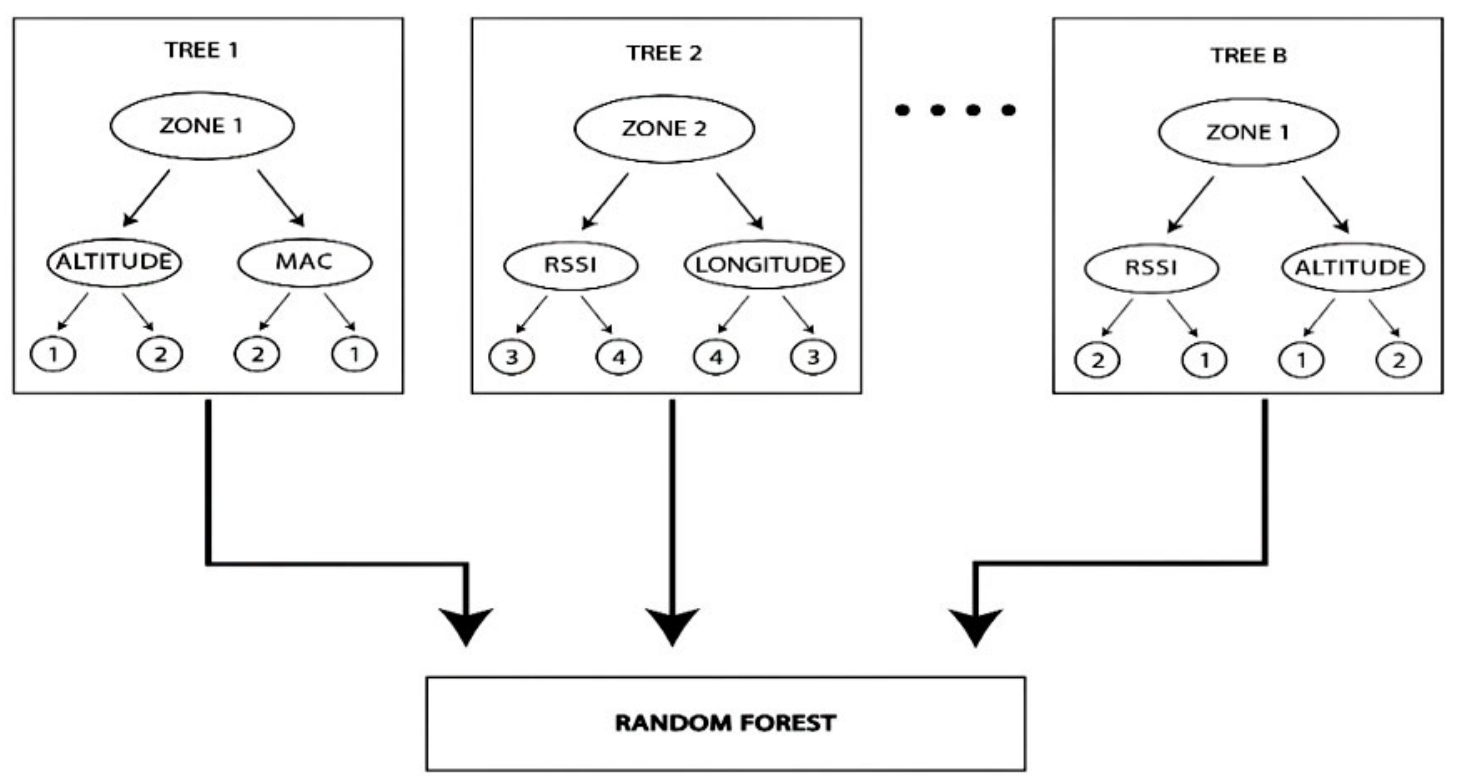

Fig. 4. RFA for aggregating output decision trees.

The operation of the RFA classifier can be summarized as follows. Suppose we have the training set $D=\left(x_{i} y_{i}\right), i=1, \ldots, n$, with $x_{i} \in \Re^{d}$. Then,

1) RFA generates the bootstrap samples $D_{b}$ from the training set $D$ with replacement where $b=1,2, \ldots, B$.

2) For each $D_{i}$, RFA constructs a classification tree $T_{i}$ and grow it until the minimum node is reached. At each node, the RFA chooses the random subset of features $m(m<d)$, and only consider splitting at those features.

3) Let $C^{b}(x)$ denote the class prediction of random forest tree $T_{b}$ by $b_{t h}$ tree. Then, the RFA prediction is given by the following majority vote:

$$
C_{R F}^{B}(x)=\operatorname{argmax}(x)\left\{\Sigma_{b=1}^{B} I\left[C_{b}(x)=g\right]\right\} .
$$

\subsection{Numerical and Experimental Results}

In this section, we apply the machine learning technique to the larger amount of data captured from the Wi-Fi Pineapple and the UAV GPS, and then, present numerical results on the accuracy of the RFA for the considered scenario. 


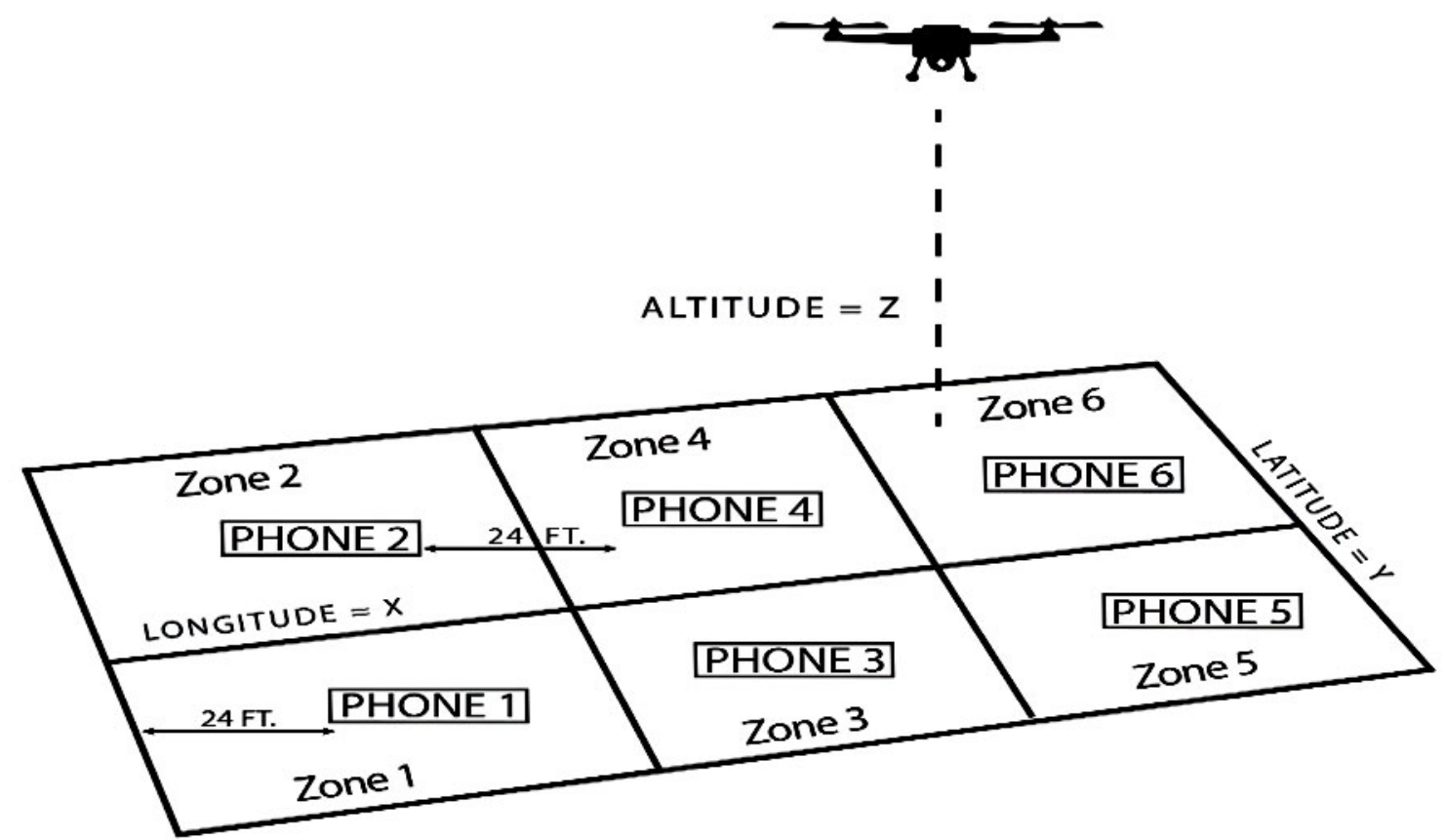

Fig. 5. Classification of Wi-Fi devices into different zones using the longitude, latitude, altitude, and RSSI captured at UAVs.

\subsubsection{Device Localization Using RFA}

The main goal of our experimentation is to accurately associate a Wi-Fi device with the correct zone that provides a coarse location information about the Wi-Fi device's location. We deploy six different phones at specific locations (zones) and have the arrangement, as displayed in Fig. 5. We divided our region into six zones and labeled them, as $y_{n}$ where $n \in\{1,2,3,4,5,6\}$. The partition design is chosen to allow the UAV flying at any given zone to capture Wi-Fi probe requests from adjacent and nonadjacent regions. The design permits the creation of many decision trees chosen from any given zone and even many trees from the same zone, and each has a different subset of features to create the random forest.

\begin{tabular}{|c|c|c|c|}
\hline Device 1 & 216 & Device 2 & 408 \\
\hline Device 3 & 407 & Device 4 & 499 \\
\hline Device 5 & 110 & Device 6 & 170 \\
\hline
\end{tabular}


TABLE II. Size of the training data for each device.

\begin{tabular}{|c|c|c|c|c|c|c|c|}
\hline Actual & \multicolumn{7}{|c|}{ Predicted } \\
\hline & Dev. 1 & Dev. 2 & Dev. 3 & Dev. 4 & Dev. 5 & Dev. 6 & $\begin{array}{c}\text { Class } \\
\text { error }\end{array}$ \\
\hline $\mathbf{1}$ & 183 & 8 & 9 & 13 & 0 & 2 & 0.1488372 \\
\hline $\mathbf{2}$ & 2 & 340 & 36 & 20 & 2 & 8 & 0.1666667 \\
\hline $\mathbf{3}$ & 6 & 30 & 337 & 20 & 3 & 5 & 0.1596010 \\
\hline $\mathbf{4}$ & 7 & 20 & 15 & 440 & 6 & 10 & 0.1164659 \\
\hline $\mathbf{5}$ & 2 & 9 & 13 & 19 & 61 & 5 & 0.4403670 \\
\hline $\mathbf{6}$ & 2 & 16 & 10 & 20 & 2 & 119 & 0.2958580 \\
\hline
\end{tabular}

TABLE III. Confusion matrix constructed using captured data.

Let $\mathrm{D}$ denote the total number of observations captured by the UAV, where we have $D=$ 1810 for our experiment. The training data set is distributed among the six devices, as shown in Table II. Out of $D$ observations, $D_{i}$ sample cases are chosen at random, to start training the data and constructing multiple trees. In this experimentation, each tree will have a random selection of variables as input, having a different set of features chosen from the data. For instance, zone-1 may have predictors chosen at random, having Phone1 and Phone2 and using a random subset of features of Altitude and MAC address to create TREE1, while another tree TREE2 may have predictors chosen at random from zone-2, having Phone3 and Phone4 and, using a random subset of features of RSSI and Longitude. The process will continue to create randomness into the model by considering the subsets of distinctive features falling into different zones.

The algorithm shows how a combination of decision trees are constructed using the bootstrapping technique with replacement. The five attributes utilized in the method are 
longitude(x), latitude(y), altitude(z), RSSI, and MAC Addresses, which are randomly selected from the best possible split among the variables. For each split, the best split among all attributes are chosen. The number of attributes to search at each best possible split is 2 , which is determined by taking the square root of the total number of the 5 attributes used in this experiment. Using bootstrapping techniques for classification experiments and taking the square root of the total number of attributes or features are preferred. The process continues until a complete random forest is created by displaying the data in a confusion matrix, as depicted in Table III. Since RFA is capable of handling a significantly large amount of data as input variables, the method is utilized by experts in categorical classification as presented in this thesis.

\subsubsection{Confusion Matrix}

The data is displayed using a confusion matrix, as shown in Table III. The matrix is composed of zones, with each row representing the original classification of the test devices arrangement on the ground and the columns representing the predicted classification. In Table III, the diagonal elements in the confusion matrix signify the number of Wi-Fi probe requests captured and correctly identified to the test device on the ground. For example, consider the diagonal element $[1,1]=183$ of the matrix, the value of 183 implies that the number of captured Wi-Fi probe requests belong to device\#1, during the original and generated classification. Whereas, the nondiagonal matrix element represents mismatched classification falling under a different test device. For example, matrix cell $[1,2]=8$ implies the Wi-Fi probe requests were incorrectly classified under device\#2 instead of device\#1.

The last column in the confusion matrix shows the class error for each of the six classified devices on the ground. Intuitively, we can conclude that the UAV correctly captured and mapped the bulk of the classification generated from the original Wi-Fi probe requests to the test devices on the ground. For example, the device $\# 2$ has a class error of 0.1666667 , indicating that 
approximately $16 \%$ of the originally captured Wi-Fi probe requests from the device will not show up in the classification generated. However, an interesting advantage of having the data tabulated in a confusion matrix is the overall accuracy of the classification model. In the case of device \#5 and \#6, a higher class error is obtained, as compared to the other devices due to a smaller size of the observation data. The smaller size of the observation data is due to the captured probe requests not being part of the classification generated, negative GPS coordinates recorded for the captured Wi-Fi probe requests, or the interval at which the device transmits the Wi-Fi probe requests.

\subsubsection{Results Analysis}

In this experimentation, we observe that the prediction of the random forest has an $81.8 \%$ accuracy. Overall, the accuracy describes how often the performance of the classifier is correct. We use the following equation to determine the overall accuracy of the method:

$$
\text { Accuracy }=\frac{N_{T P}+N_{T N}}{N_{\text {Total }}},
$$

where the number of true positives (TP) $\left(N_{T P}\right)$ is the number of occurrences when the model predicts the correct probe requests captured in the correct cell, which are found by adding all the diagonal elements in the confusion matrix, as depicted in Table III. The number of true negatives (TN) is denoted by $N_{T N}$, which predicts correctly the probe requests captured not falling in the correct cells and are found in the row elements of the confusion matrix, except for the diagonal elements, as illustrated in Table III. The $N_{\text {Total }}$ is the number of all observed probe requests captured during the experiment, as shown in Table II.

This implies that there is an $81.8 \%$ chance that the phone location on the ground is correctly identified in the zone where the Wi-Fi device is located, as illustrated in Fig. 5. However, if 
precision and recall for the entire classification model are to be computed, the model does show larger gains. In particular, the precision can be calculated as:

$$
P=\frac{N_{T P}+N_{F P}}{N_{\text {Total }}},
$$

where $N_{F P}$ is the number of false positives (FP), which classify data in the model, as the Wi-Fi probe requests captured falling in the correct cell classification generated but falling in the wrong cell. FPs are the row elements in the Confusion Matrix. For this experiment, the predicted and generated Wi-Fi probe requests captured by the Wi-Fi Pineapple belong to the same test device on the ground. The precision model has an $82 \%$ precision classification, implying the predicted WiFi probe requests coming from any of the six test devices is matched correctly to the generating test device.

On the other hand, the recall for the RFA is calculated as:

$$
R=\frac{N_{T P}}{N_{F N}},
$$

where $N_{F N}$ is defined as the number of false negatives, which classify WiFi probe requests captured, as falling in the wrong cell classification but having actually falling in the correct cell. The false negatives (FNs) are found in the column elements of the matrix. The model has an $80 \%$ recall classification, implying the predicted Wi-Fi probe requests from any of the six test devices is matched correctly to the generating test device. This model shows a strong correlation between precision and recall, demonstrating how accurate the model is in predicting the location of the test devices on the ground grid, as shown in Table III. 


\section{CHAPTER IV}

UAV Procedures to Extend Flight Time for Search and Rescue Operations Using Wi-Fi Probe Requests

\subsection{Environment of Experimental Design}

\subsubsection{Sensor to Detect Wi-Fi Probes}

The sensor used in this research to detect Wi-Fi probe requests is called Wi-Fi Pineapple Tetra, which is a Wireless Auditing Arsenal [50] that is used for Wireless Penetration Testing. Unlike the Wi-Fi Pineapple previously discussed in section 3.1.1, the Tetra is bigger. Therefore, it has a more extensive footprint, and the antennas are upgraded to a $5 \mathrm{dBi}$ range, increasing the coverage area. The Tetra dual-band $(2.4 / 5 \mathrm{GHz})$ capabilities provide the solution to the continuous detection of probes in either band. Tetra is powered up by a DC battery, providing its own entirely independent power and does not strain the battery of the UAV. Due to its design implication for portability, long-term deployment, and on-the-move auditing, Tetra is ideal for mounting by straps at the bottom of the UAV frame. The weight difference compared to the Wi-Fi Pineapple, the architecture of the Tarot 650 is able to handle the added payload of the Tetra when flying over the designated search area detecting probes. For our research, to establish a secure connection between the Laptop and Kali Linux to code the Tetra, we used a Virtual Private Connection and PuTTY. PuTTY is an open source software that is used as an SSH client for the Unix Machine. For collecting data, we used a virtual machine application Kali Linux VMware to operate the Tetra relative to the ground base station. The Kali Linux contains pre-install applications, including Wireshark, an application that analyzes packets [52]. Each probe detected is synced to the UAV flight controller to obtain the GPS coordinates in a 3-D format from the telemetry logs obtained from the ground base station. 


\subsubsection{DroneDeploy}

DroneDeploy is a software platform for UAVs to create a real-time mapping of an area of interest and to provide information about the planned flight [53]. The planned flight of the UAV includes the entire search area, the total time based on the flight pattern, the altitude to fly the mission, and the number of batteries required to complete the planned flight. DroneDeploy works great in scanning and creating SAR flight patterns of the search area without having to deploy the UAV.

In this work, DroneDeploy is used to create different search patterns for the UAV to fly autonomously and compare the results to piloting the UAV remote-control. Each search pattern takes different total time and has a different effect on the battery consumption of the UAV. The variation of probes identified is based on the search pattern flown by the UAV.

\subsubsection{FAA Rules and Regulations for UAVs}

There are a set of rules outlining safety guidelines for operators to follow when flying a UAV that is set by the Federal Aviation Administration (FAA) [54]. The FAA is the agency responsible for overseeing the safety of civil aviation. Operators must maintain the UAV within visual line of sight, i.e., they must be able to see the UAV with their naked eyes, and fly it only in the daylight. The maximum altitude to keep the UAV is under $400 \mathrm{ft}$. from the ground, and it cannot be flown over people or nearby buildings. Due to the UAV flight tests conducted for this thesis in an area the size of about two acres and without the involvement of any pilot using the fully autonomous capabilities of the UAV, the operator must fly following the FAA guidelines to keep any damages, if any were to occur, to a minimum. To assist the operator in determining whether there is any flight restriction at the location where the UAV is operated, the B4UFLY smartphone app is used. All FAA safety guidelines are followed during all flight tests conducted for this study. 


\subsubsection{UAV Used for the Experiment}

Given the application of this experiment, a rotary-wing UAV is used for its capabilities of vertical takeoff and landing, capacity to hover at specific locations as set by the autonomous function or by the pilot when flying in $\mathrm{RC}$ mode and perform a variety of flight maneuvering. The UAV is a quadcopter, having a Tarot 650 frame. The Tarot 650 is a lightweight and sturdy frame made up of carbon fiber material. The Tarot 650 is equipped with four $620 \mathrm{kV}$ high power brushless motors, 30-amps Electronic Speed Controllers to supply the amperes controlling the speed of the brushless motors, 13-inch carbon fiber propellers, and a 4S 4000 mAh Lipo-battery, which provides between 15 and to 20 minutes of quality flight time. The payload capacity of the UAV is about $2.5 \mathrm{~kg}$ and a 3-4 kg takeoff weight. The Tarot 650 quadcopter is equipped with a power distribution board (PDB) to distribute power efficiently to all the components on the UAV. The PDB is a crucial component of the UAV, and if it is not chosen and configured the right way, the UAV could end up crashing to the ground. The PDB is a circuit board that connects the LiPo battery to all the ESCs

on the UAV. Furthermore, the Pixhawk autopilot is positioned at the top plate of the frame and connected to an external GPS module away from the different components of the UAV, avoiding or keeping to a minimum any interference from the motors, propellers, or sensors. The GPS module connects to the GPS satellites to receive precise navigational data for Stabilize and Auto flight mode.

\subsection{Search Theory Method}

In this paper, we use the search theory method of probability that has been utilized for SAR operations since WWII [55]. Applying its mathematical foundation to SAR operations, the theory has shown to be valuable by optimizing the allocation efforts to designated search areas and increasing the likelihood of detecting the lost subject in the least possible amount of time. This method is chosen for its practical applications to search planning techniques and for its 
effectiveness in increasing the probability of the overall search success rate. Moreover, the study extends the concept of search theory used in the ground to the air, using a UAV and the Wi-Fi Pineapple Tetra as the sensor to detect the search object(s).

There are uncertainties in any SAR mission, i.e., what areas to begin searching in any given terrain to maximize any Wi-Fi probe requests detection. Other types of uncertainties include how the weather may impact the UAV itself, the Wi-Fi Pineapple Tetra sensor mounted onto the UAV to detect probes, and any foliage, which might be covering the phones on the ground. Considering and understanding these uncertainties quantitatively using probability theory techniques will maximize the overall chances of a successful SAR mission.

\subsubsection{Probability of Containment}

The amount of area to cover during SAR missions is limited to maximize the chances of finding the search object quickly. After all available facts have been gathered, a search area is determined, providing the possible location of the search object. This process is known as the probability of containment (POC). The POC not only determines the possibility area by eliminating the less likely areas not containing the search object but also divides a larger area into sub-areas since the amount of area to efficiently cover is limited. Sensor technology is used on UAVs to carry out one or more tasks during SAR missions, and the measure of how well the sensors performed undertaking the SAR missions is known as the probability of detection (POD).

\subsubsection{Probability of Detection}

Factors that affect the probability of detection (POD) are the type of terrain, the type of search object or the detection of probes, and the type of sensors use. POD performance is measured by the ability to detect the search object only if the search area includes the object. Based on probability theory, this concept is known as conditional probability. By definition, conditional 
probability explains the probability of an event occurring given the previous or other events have already occurred.

An area can be searched under different circumstances giving a different probability of detecting the search object. The likelihood of finding the search object is high if the object is in the area at the time of the search. During the search, the POD predicts how well the sensors perform or how thoroughly an area has been searched. In some SAR cases, an area may have to be searched multiple times to increase the likelihood of detecting the search object. Neither the POC nor the POD stand-alone is a valid measure to predict the chances of success during a search. The probability of success (POS), however, is an accurate measure to predict the chances of success during a search.

\subsubsection{Probability of Success}

The POS is defined as the probability of finding or detecting the search object in the designated search area. The POC and POD determine the value of POS. The POS can be expanded using probability theory notation and expressed as:

$$
P(A \cap B)=P(A) \cdot P(B \mid A),
$$

where event $\mathrm{A}$ is the search object in the designated area to be searched; event $\mathrm{B}$ is the sensor used to detect the search object given a designated area to be searched; $\mathrm{P}(\mathrm{A})$ is the probability that the search object is in the designated search area (event $\mathrm{A}$ ) or $\mathrm{POC}$ will be true; $\mathrm{P}(\mathrm{B} \mid \mathrm{A})$ is the conditional probability that the sensor detects the search object in the designated search area (event B) or POD will be true, given that the search object is in the search area; and $\mathrm{P}(\mathrm{A} \cap \mathrm{B})$ is the probability that during a particular search (POS) the search object is located in the designated search area. 
Predicted values for POC and POD are based on factors, such as what are the actual conditions in the designated search area, what amount of the selected area is covered, and what is the most up-to-date data analysis from all the gathered information. Then, an estimated value of the POS can be determined. The POS is only an estimated value for which the actual value cannot be determined, and the value may have to be adjusted for the POS as newer data comes to light. When the value of either the POC or the POD is updated, the POS needs to be adjusted. By searching an area and not detecting the search object, the probability of POD is reduced by an amount proportional to its predicted value.

\subsection{Ways to Maximize UAV Flight Time 4.3.1 Optimization of Propellers}

A UAV system integrates many components to optimize its flight performance. However, the flight controller (FC) or autopilot is considered the most important component of the UAV. The FC functions as the brain of the UAV, managing all of its functionalities. The FC is not only composed of hardware but also of the software comprising of advanced programs and mathematical algorithms. One of the sources connected to the FC is the Inertial Measurement Unit (IMU).

The IMU hosts the accelerometer and gyroscope sensors. Advanced FCs come with 6-axis accelerometer/gyroscope. An accelerometer measures the orientation of the UAV relative to the earth's surface, while the gyroscope measures the rotational force. The IMU combines these two measurements for the flight controller to be able to calculate the angle at which the UAV is flying and performing the necessary adjustments to improve the UAV flight capabilities.

The three principal axes that control the motion of a UAV are Pitch, Yaw, and Roll. The forward or backward movement of the UAV is the function of Pitch. The forward Pitch makes the UAV to tilt slightly in the forward direction and to move away from the pilot, and the backward 
Pitch brings the UAV back to the pilot. Yaw controls the rotation of the UAV either to the left or the right, while Roll controls the sideward flying of the UAV either to the left or the right.

A UAV will undergo continuous changes in acceleration during a flight trajectory. The IMU 6-axis accelerometer sensor is responsible for detecting changes in the current rate of acceleration by using one or more of its accelerometers. The IMU also detects changes in Pitch, Yaw, and Roll making use of one or more of its gyroscopes. The IMU calculates the UAV's current velocity by integrating its acceleration and gravity.

UAVs experience natural vibration during flight, and vibration is one of the overlooked challenges for UAVs. A primary source of vibration in a UAV is due to the propellers being out of balance. Hence, balancing the UAV's propellers is essential to have an optimal flight performance, avoiding inaccurate estimates of the UAV position by disrupting the flight controller accelerometers/gyroscopes, and preventing the LiPo battery from draining faster. The IMU can negatively be affected by the excessive vibration from unbalanced propellers.

The accelerometer sensors have a threshold to resist vibration frequencies during a flight. Exceeding the sensor threshold by the frequency of the vibrations is illustrated in Fig. 6. Inside the sensor's range, the IMU sends accurate data of the position and the altitude of the UAV. The highfrequency vibrations produced by the propellers outside the sensor's range generate discrepancies between the calculated position and the actual position [30]. As the throttle is applied to increase the UAV speed, the motors spin faster causing propellers to turn faster and increasing vibration, which produce a drift or divergence in the attitude estimation of the UAV. The software parameters to control and 


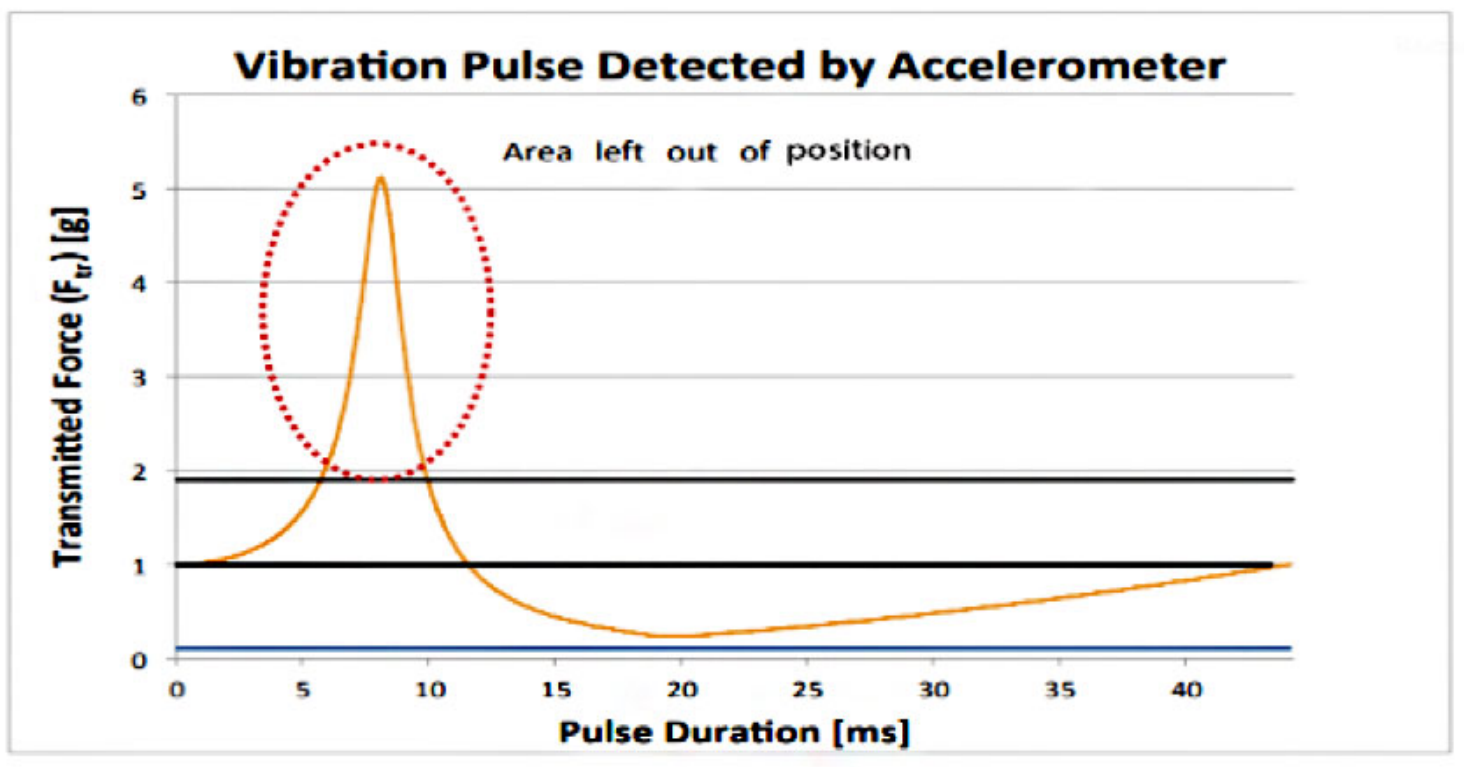

Fig. 6. Accelerometer sensors' threshold to resist vibration [56].

adjust the UAV IMU cannot always fix the vibration problems. The balancing of propellers to prevent or to minimize vibrations must be performed as a pre-flight UAV safety checklist to enhance performance and increase the flight time, which are two crucial elements to conduct SAR operations.

\subsubsection{LiPo Battery Basics}

The primary source to power up a UAV is the battery. For UAVs, the ideal type of battery is the Lithium Polymer (LiPo) due to its lighter weight, high power, and fast and high discharge rates. Extended flight time and performance of the UAV are dependent on the LiPo capacity, while at the same time not adding scaling problems to the positive correlation between LiPo capacity and weight. The bigger the LiPo capacity, the heavier the LiPo becomes, which might not be efficient since a heavier LiPo will require lower motor $\mathrm{KV}$ ratings operating at higher voltage ranges. The addition of weight to the overall 


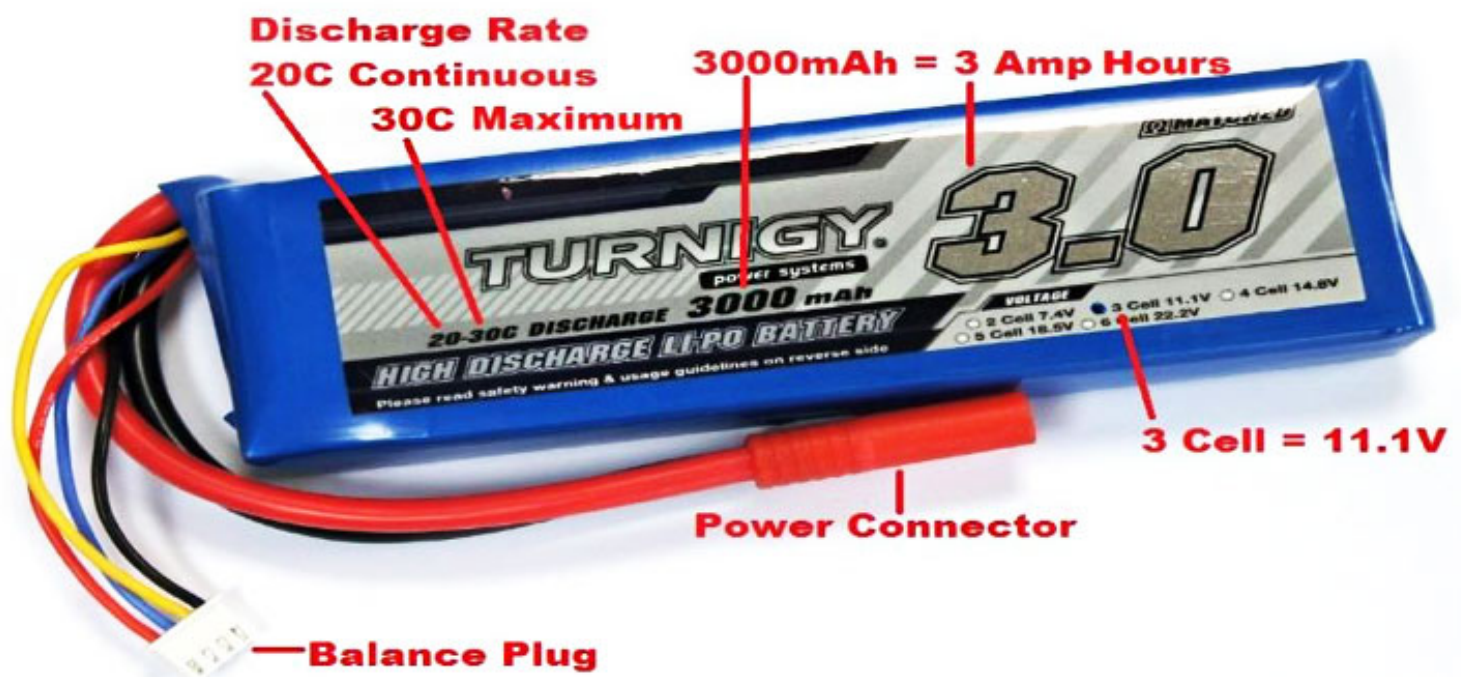

Fig. 7. LiPo battery functions [57].

payload of the UAV affects the duration of the flight time and navigational performance. The LiPo battery needs to fit within the frame of the UAV. The size of the LiPo will depend on the size, type, and the number of motors of the UAV. Understanding how to read the specifications on a LiPo, as illustrated in Fig. 7, means a longer flight time, better maneuverability, and a faster flying. LiPo batteries are made up of cells in series ranging from $1 \mathrm{~S}$ to $6 \mathrm{~S}$. These cells specify the LiPo voltage. Each cell is composed of a nominal voltage of $3.7 \mathrm{~V}$. Nominal voltage is a term manufacturers have come up with to designate a middle safe voltage range. As shown in Fig. 7, the LiPo has three cells and an $11.1 \mathrm{~V}$, which is the nominal voltage calculated by multiplying the number of cells by $3.7 \mathrm{~V}$. The UAV motors' RPM is directly 


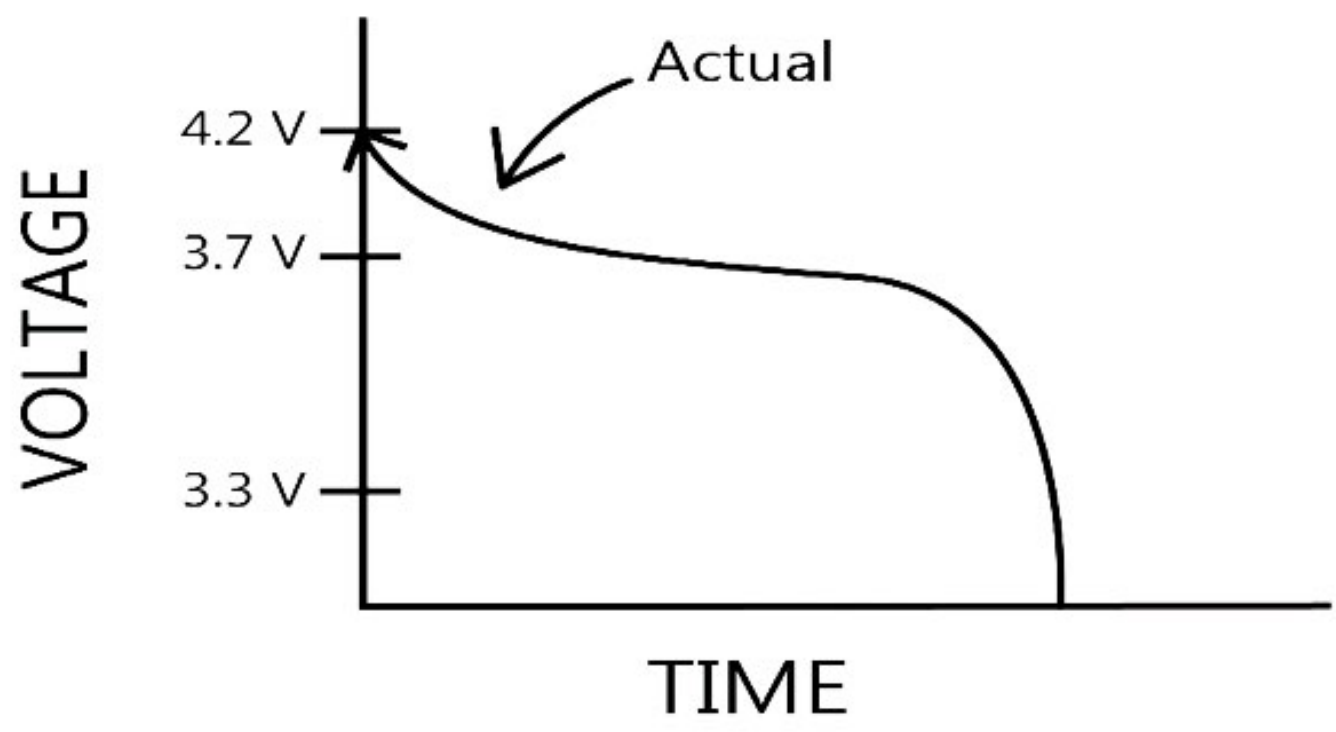

Fig. 8. Actual LiPo battery discharge curve [58].

proportional to the voltage supplied by the LiPo. A higher cell count LiPo is capable of providing faster spinning in the motors and ESCs if these components support a higher voltage.

LiPo batteries operate in a $4.2 \mathrm{~V}$ when fully charged and safely discharged when they reach the cutoff voltage of a $3.0 \mathrm{~V}$. Either charging it above a $4.2 \mathrm{~V}$ or below the cutoff voltage can cause the LiPo to explode or permanent damaging it. Discharging the LiPo below a 3.0V will also negatively affect the flight performance of the UAV by diminishing the performance levels of the LiPo and shortening the UAV flight time. The UAV needs to have sufficient time to return to its home position $(\mathrm{RTH})$, which is the position where the UAV was armed to takeoff. A cutoff voltage of a $3.3 \mathrm{~V}$ is used instead of a $3.0 \mathrm{~V}$ to compensate for the distance the UAV needs to RTH. Setting the right voltage cutoff and the process of discharging functions in a LiPo is critical to understand to get the maximum flight performance of the UAV.

Fig. 8 illustrates an actual case showing how a LiPo is discharged during use. An actual Lipo discharging process starts at its maximum charging voltage of a $4.2 \mathrm{~V}$. Then, it proceeds to discharge slowly, as the UAV continues its flight trajectory, drawing current from the LiPo until 
reaching the cutoff point of a $3.3 \mathrm{~V}$, which is the safest level of discharging, as previously explained. In Fig. 8, a cutoff voltage of a 3.3V is used, but not all LiPo batteries are manufactured the same. Depending on the brand, each LiPo will need to be set at either a $3.0 \mathrm{~V}$ or a $3.3 \mathrm{~V}$ as the cutoff value based on the manufacturer's datasheet or by performing actual flying tests of the UAV to determine the value.

Another LiPo feature to understand is its capacity. From Fig. 7, capacity is indicated by the value of 3000mAh or a 3 Amp Hours measured in Amp-hrs or milliamp-hrs. Larger values allow the LiPo to have a more extensive capacity to store more of its total energy to last longer during a flight. The $3000 \mathrm{mAh}$ LiPo will take an hour to discharge from the maximum voltage to the cutoff voltage. The 3 Amp Hours current is provided by the LiPo for one continuous hour if a constant current of a $3 \mathrm{Amp}$ is drawn for one hour, but it is not the actual case since LiPo batteries do not discharge at a constant voltage rate, as depicted in Fig. 8.

Increasing the capacity of the LiPo makes the LiPo heavier and larger, but a longer flight time can be attained. This positive correlation is only accurate to the point at which the UAV flight time becomes ineffective, as depicted in Fig. 9, e.g., and remains constant, even after the LiPo gets larger and heavier. Choosing a larger LiPo capacity to increase the UAV flight time is not a practical approach to follow most of the time. The procedure is only adding a more substantial payload to the UAV. Consequently, the UAV will need to draw more current from the LiPo and will reduce the amount of flying time. Calculating the estimated flight time of a LiPo can be done by conducting flights under different conditions, e.g., windy condition and unusual flight pattern, to get a sense of how much current the battery is drawing or checking the manufacturer's datasheet if it is reliable.

Another misconception about capacity is that if the discharged current is double from a 3 Amp Hours to a 6 Amp Hours, flight time, while keeping the cutoff voltage at a 3.3V, will be 
reduced by half. This capacity only applies if the assumption is made that the UAV is drawing a constant current from the LiPo based on a steady hover. However, in an actual case, the UAV experiences different speeds at which the motors spin due to the wind speed. The UAV needs to make the proper adjustment by using its stability sensors, which will pull current at a non-constant rate. Consequently, the higher current draw will reduce the flight time by more than a half. Depending on the LiPo, manufacturers provide datasheets of discharge curves. Most of them are based on a constant draw current, as in this example of a 3 Amp Hours, for a continuous hour. To make a better assessment of an estimated discharge rate is to test the aircraft under different conditions to make sure how the aircraft will perform during a critical SAR operation beforehand.

Higher capacity also gives out higher discharge current, which is known as the C-rating. The C-rating is another critical feature of a LiPo to understand. It relates to the maximum amount of Amps a LiPo can safely deliver at a continuous and burst (maximum) discharge rating. In Fig. 7, 20C and 30C are shown, respectively. 20C represents the continuous discharge rating delivered consistently by the LiPo while maintaining a stable dropping voltage. The safe maximum current draw is calculated as:

$$
I C=C \cdot R C=3000 \mathrm{mAh} \cdot 20 \mathrm{C}=60 \mathrm{~A},
$$

where IC is the continuous current draw, $\mathrm{C}$ is the capacity of the battery, and RC denotes the Crating. The result of a $60 \mathrm{~A}$ is the maximum continuous safe load handled by the LiPo. The other value of a $30 \mathrm{C}$ is the burst current, and it is the maximum current a LiPo can push out for about 10 seconds under full throttle and dropping voltage rapidly. The burst current is defined as:

$$
I_{\max }=C \cdot R B=3000 m A h \cdot 30 C=90 A \text {, }
$$


where Imax refers to the maximum draw current, and RB refers to the burst current. How to determine the right C-Rating needed for a UAV is based on the type of the UAV. The number of propellers determines the type of a UAV. For example, a four-propeller UAV is called a quadcopter. Four is multiplied by each of the motors drawing current under load found in the datasheet specifications of the manufacturer. The combined draw current of all the four motors cannot exceed the safe maximum current draw by the LiPo, as illustrated in Fig. 7 and 8.

Having a necessary and proper understanding of LiPo battery specifications is essential to get the most efficient and longest flight performance of a UAV. The SAR operations are critical and required fast action and reliability to deploy a UAV to search and rescue victims.

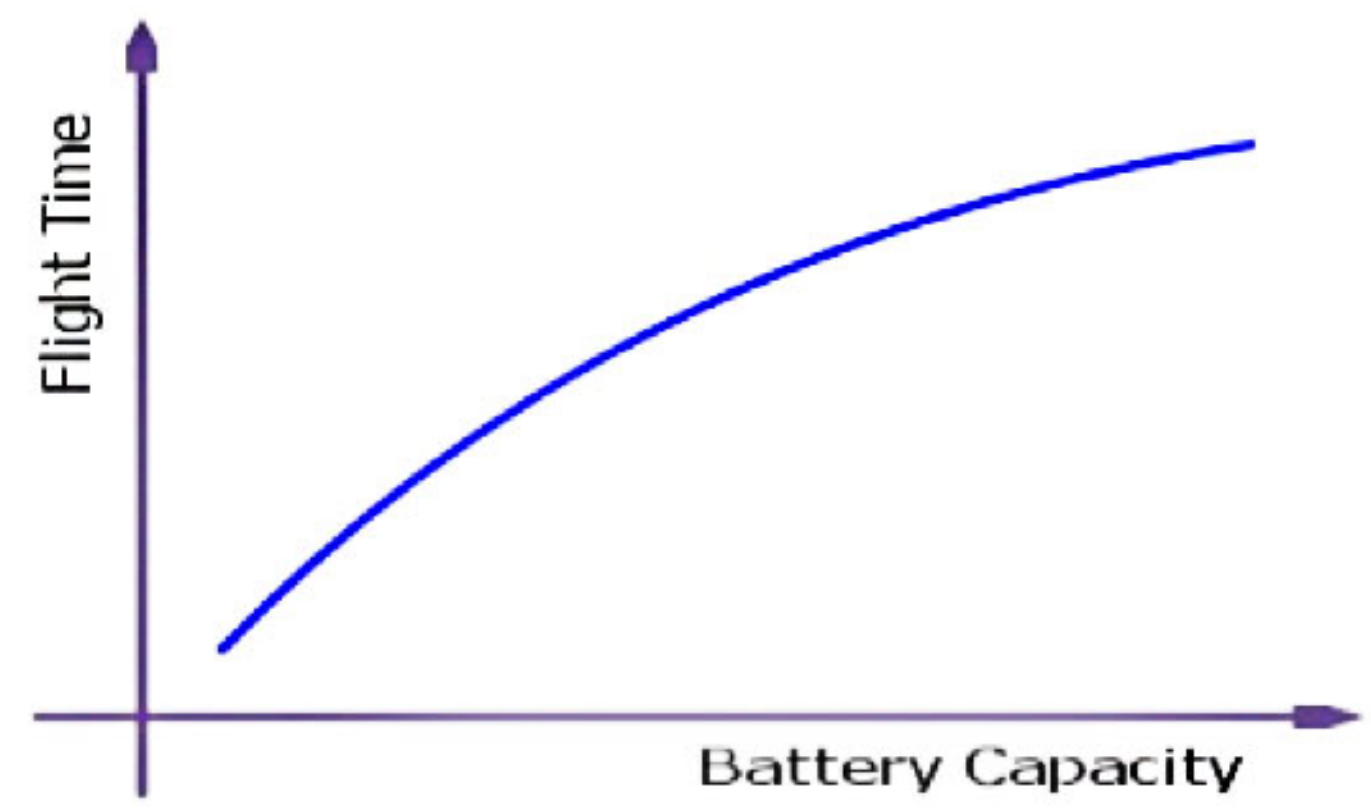

Fig. 9. Relationship between flight time and LiPo capacity [59].

\subsection{SAR Flight Patterns Followed by UAV Affect Optimization of UAV Flight Times}

Instructions about what type of search patterns to incorporate into a flight plan are set by the International Maritime Organization and International Civil Aviation Organization. These search and rescue flight patterns are published in the International and Aeronautical and Maritime Search 
and Rescue manual (IAMSR) [60]. As explained in the IAMSR manual, the search and rescue flight plans have their own characteristics and conditions, under which they are best suited to perform. One fundamental question for the task forces, specializing in search and rescue to consider before deploying a UAV, is how to optimally plan the UAV flight path to achieve the operational goals, considering the search area and sensors' range limitations. In this section, the flight plan describes the different search and rescue flight patterns that suit the designated search areas of various sizes and shapes. The

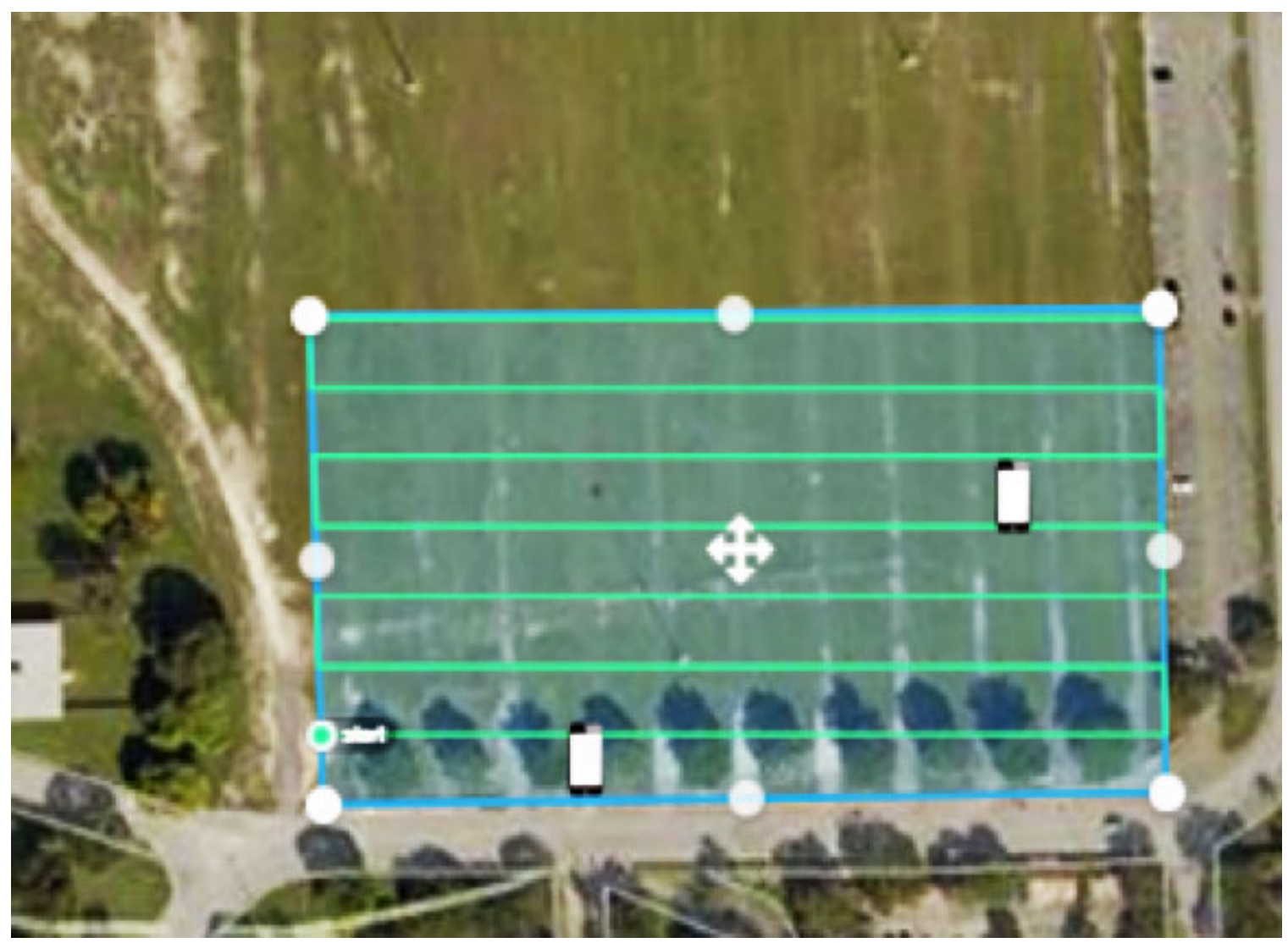

Fig. 10. Parallel Track Search Pattern traveled by the UAV.

different flight patterns consume the UAV battery life differently. These flight patterns are probably the most commonly used during SAR missions, executed by airplanes, helicopters, and UAVs. A description of how the UAV LiPo power consumption is affected by each of the three flight patterns tested is presented. 


\subsubsection{Parallel Track Search Pattern (PTSP)}

As illustrated in Fig. 10, the UAV follows the long tracks straightforward. At this stage, the UAV performs the horizontal forward movement, maintaining a steady altitude of about 85 feet. The forward motion converts the takeoff air, which is vertical air, mainly onto horizontal airflow, allowing the incoming wind to enter the rotor system and improving the

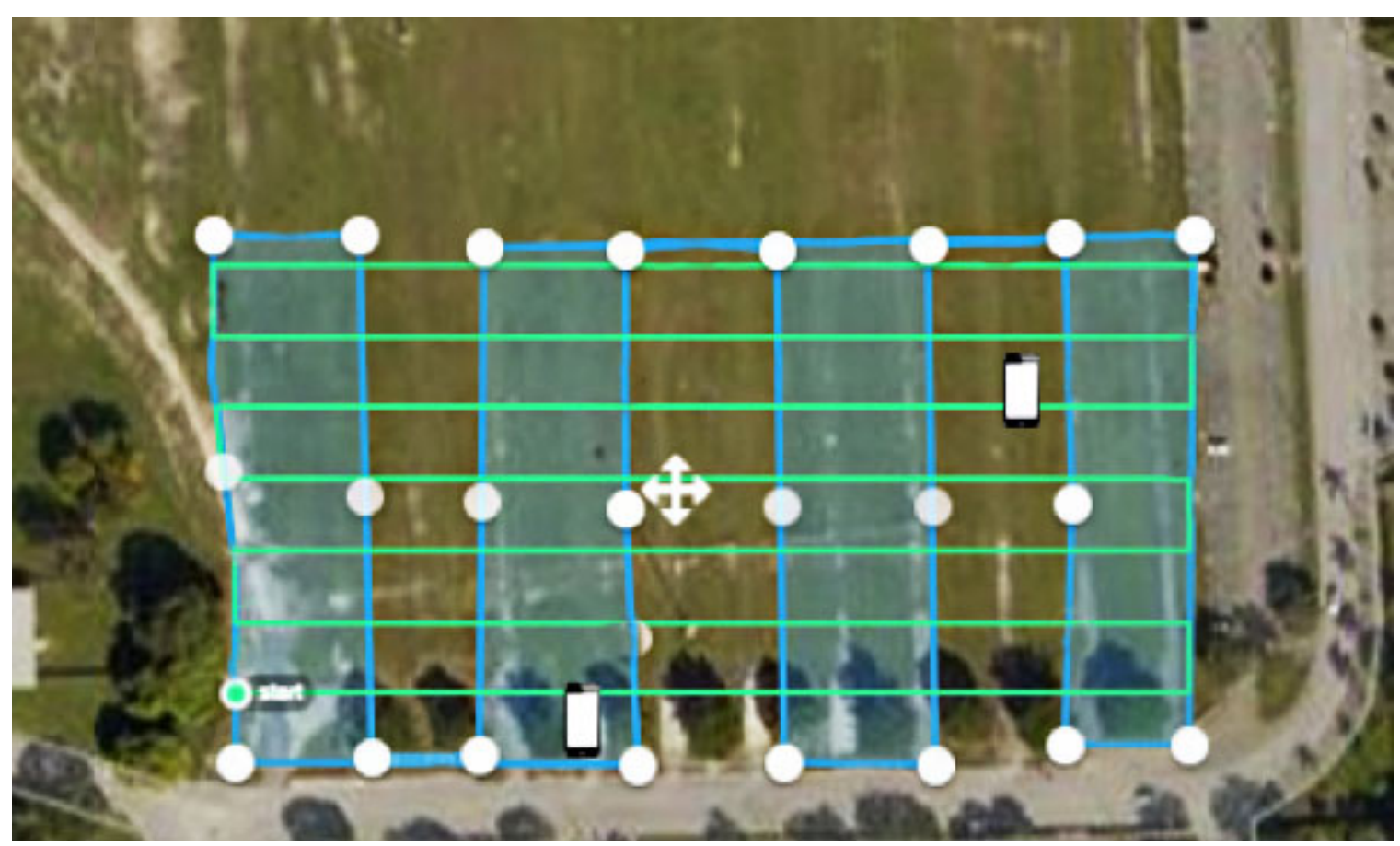

Fig. 11. Creeping Line Search Pattern traveled by UAV.

flight efficiency of the UAV. How propellers react to the changing induced airflow? The airflow becomes more horizontal and increases the propellers angle of attack, continuing to add more efficiency to the UAV flight. The term used to describe this added efficiency is called the translational lift. The forward motion causes efficiency in the rotor system, improves the UAV performance, and increases the LiPo flight time.

Approaching each of the turns for the short tracks, the UAV performs either a hover for a few seconds at each waypoint and then continue its pre-programmed waypoint flight path or continue on with the mission, passing and turning at each of the waypoints without stopping. 
Hovering at each waypoint is avoided since it requires the UAV to draw more current from the LiPo to maintain its thrust stability. The UAV flight plan is to take advantage of translational lift from the very takeoff to completing the Parallel Track Search Pattern at landing.

\subsubsection{Creeping Line Search Pattern (CLSP)}

The Creeping Line Search Pattern is similar to the PTSP, except that the search legs, which are the longest track in the search pattern, are parallel to the shortest side of the area to be searched, as illustrated in Fig. 11. The UAV flight plan is in a vertical orientation, following the search legs and turning without stopping at the short tracks where the waypoints are set. Once the UAV has reached a waypoint, the rotation is not set in a tight or quick turn radius to avoid any current overdraw from the battery life. The flight plan goal is only to current draw the necessary amount from the LiPo to keep it at its maximum flight time and maintain the UAV peak-flight performance by taking advantage of translational lift throughout the flight.

\subsubsection{Sector Search Pattern (SSP)}

As illustrated in Fig. 12, the Sector Search Pattern consists of three interconnected triangles. The UAV starts from any of the vertices chosen as the reference and covers each of the sides until it has completely traversed the entire flight pattern. At each of the vertices, the waypoints are set for the UAV to rotate at 360 degrees gently. The 360 degrees rotation leads to a smooth navigational performance taking advantage of translational lift. The tuning of each waypoint radius allows the UAV not to rotate before or after each point to achieve smooth and efficient turns. Therefore, the battery life is extended to its maximum flight time 


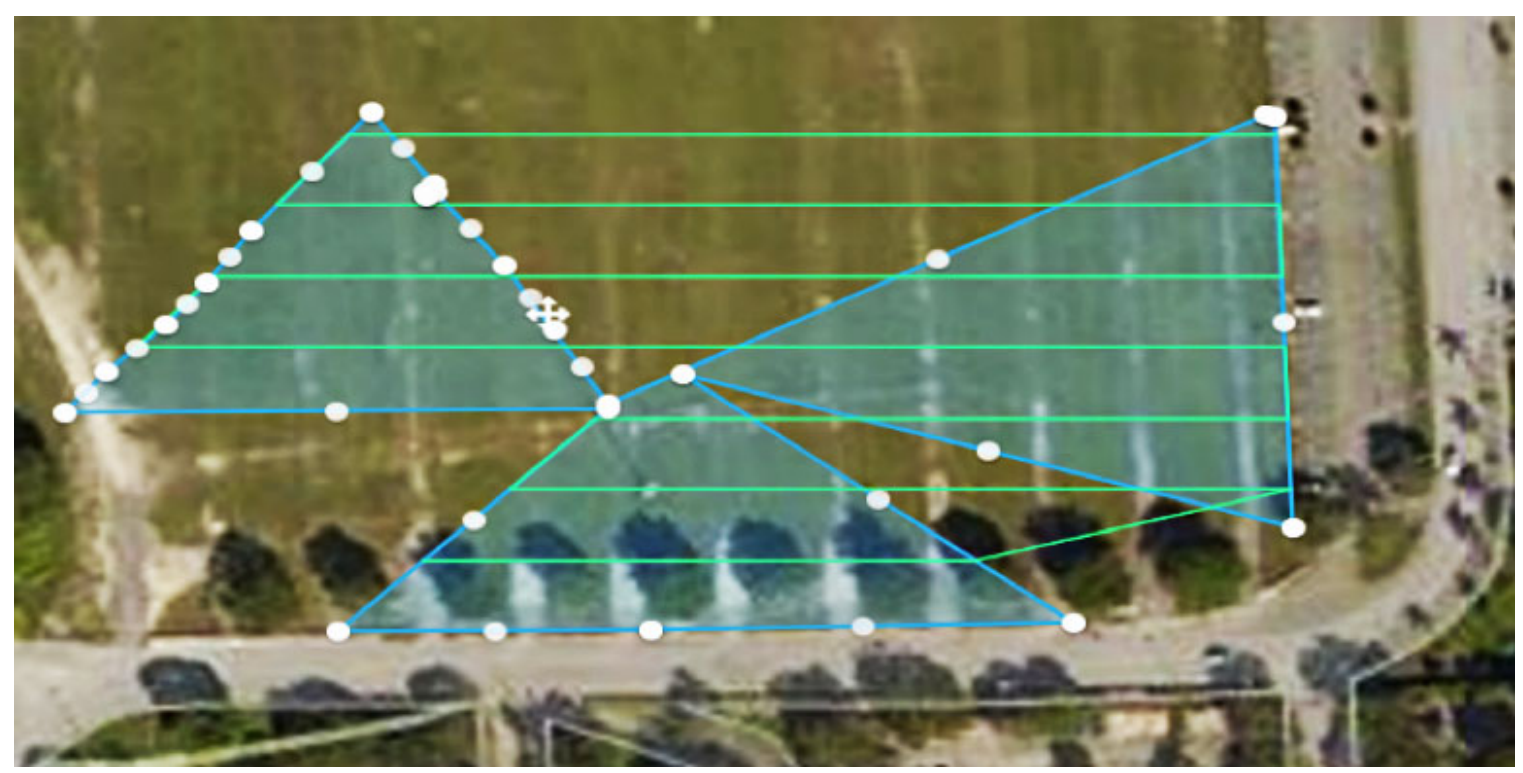

Fig. 12. Sector Search Pattern traveled by the UAV.

This flight pattern is most effective when the most probable location, which is called the datum, is small. According to Search Theory Method, a designated search area is further divided into subareas to achieve a higher probability of locating a victim or finding the search object. The same concept is used when the UAV flies over these patterns. The larger area is divided into subareas as illustrated in Fig. 12. Furthermore, only one UAV can be used for SSP flying at a similar altitude of 85 feet since the area to cover is small.

\subsection{Results Analysis}

In this section, we discuss the results obtained in real time from the UAV flying the three different SAR patterns. By setting the altitude and speed to fly the UAV over the search area, DroneDeploy determines an estimated time to complete the flight plan. The speed is set at $7 \mathrm{~m} / \mathrm{s}$, which is roughly equivalent to $16 \mathrm{mph}$. At $16 \mathrm{mph}$, the forward airspeed of the UAV becomes aerodynamically efficient since the forward speed is increasing. Beyond the $16 \mathrm{mph}$, the induced drag of the UAV forward speed prevents from obtaining any 


\begin{tabular}{|c|c|c|c|c|}
\hline $\begin{array}{c}\text { Flight } \\
\text { Pattern }\end{array}$ & $\begin{array}{c}\text { Area } \\
\text { Covered } \\
\text { (acre) }\end{array}$ & $\begin{array}{c}\text { Speed } \\
(\mathbf{m} / \mathbf{s})\end{array}$ & $\begin{array}{c}\text { Altitude } \\
\text { (ft.) }\end{array}$ & $\begin{array}{c}\text { Est. Flight } \\
\text { Time (min.) }\end{array}$ \\
\hline PTSP & 2 & 7 & 85 & 10 \\
\hline CLSP & 2 & 7 & 85 & 12 \\
\hline SSP & 2 & 7 & 85 & 9 \\
\hline
\end{tabular}

TABLE IV. SAR Flight data obtained from DroneDeploy.

benefits provided by translational lift. The altitude for the UAV to fly over the search area is set to 85 feet to allow for the Tetra sensor coverage range the ability to detect probes. Table IV illustrates the UAV flight planning report that DroneDeploy generates the calculated time required to fly the three search patterns.

The autonomous flight mission is set by using the Ground Control Station Mission Planner, as mentioned in section 3.1.2. Table V shows the data obtained by setting GPS waypoints to create the UAV flight plan, and the number of probes detected for each of the three search patterns. The altitude and forward speed are fixed throughout the UAV flight plan for each of the three SAR patterns. The SSP has the least energy consumption due to the fewer number of turns and mainly flies its path forward in a straight line. The width of all sides gets wider, leaving a larger uncovered gap inside all the three triangles.

The result is a faster coverage flight plan, but fewer probes detected. The PTSP detects the most number of probes, and it completed the flight plan with the second fastest time, having the second least number of rotations since it traverses the search area in a systematic pattern. The gaps between the long tracks are set to 40 feet, which is the horizontal coverage range of the Tetra sensor. As the UAV flies over the search area, it detects probes from adjacent and nonadjacent long tracks. It consumes about a $60 \%$ of the 


\begin{tabular}{|c|c|c|c|c|c|c|}
\hline $\begin{array}{c}\text { Flight } \\
\text { Pattern }\end{array}$ & $\begin{array}{c}\text { Area } \\
\text { Covered } \\
\text { (acre) }\end{array}$ & $\begin{array}{c}\text { Speed } \\
(\mathrm{m} / \mathrm{s})\end{array}$ & $\begin{array}{c}\text { Altitude } \\
(\mathrm{ft} \text {.) }\end{array}$ & $\begin{array}{c}\text { Time } \\
(\text { min.) }\end{array}$ & $\begin{array}{c}\text { Lipo Cap. } \\
\text { Left (\%) }\end{array}$ & $\begin{array}{c}\text { Capt. } \\
\text { Probes }\end{array}$ \\
\hline PTSP & 2 & 7 & 85 & 12 & 40 & 45 \\
\hline CLSP & 2 & 7 & 85 & 15 & 33 & 29 \\
\hline SSP & 2 & 7 & 85 & 11 & 45 & 17 \\
\hline
\end{tabular}

TABLE V. Results with an autonomous UAV over different SAR flight patterns.

LiPo, as each turn is made accordingly to the set speed to flow smoothly on each waypoint turns and avoiding aggressive maneuvers of the UAV.

CLSP consumes most of the LiPo compared to the other two SAR flight patterns. It contains the most turns. The UAV makes each of the turns smoothly by extending the long tracks. The turns are not compacting, or banking turns mostly to maintain the UAV flying at a forward speed to take advantage of translational lift. However, as shown in Table V, the more turns a flight pattern has, the more it consumes energy from the LiPo. Every turn is made in an extended and smooth manner along the short track, and the UAV trajectory is made longer, which is another reason why the CLSP discharged the LiPo more than the other search patterns tested.

Table VI shows the data collected over the search area flying the UAV in a remote-control (RC) mode. The RC mode requires skills to operate the UAV in a smooth flight style, not at full speed, and without any aggressive maneuvers to avoid unnecessary overloading in discharging the LiPo faster. Under RC mode, the UAV captured the most probes compared to any of the three search flight patterns flown autonomously. However, the trajectory took longer to complete and consumed more power from the LiPo. 


\begin{tabular}{|c|c|c|c|c|c|c|}
\hline $\begin{array}{c}\text { Flight } \\
\text { Pattern }\end{array}$ & $\begin{array}{c}\text { Area Covered } \\
\text { (acre) }\end{array}$ & $\begin{array}{c}\text { Speed } \\
(\mathbf{m} / \mathbf{s})\end{array}$ & $\begin{array}{c}\text { Altitude } \\
(\boldsymbol{f t .})\end{array}$ & $\begin{array}{c}\text { Time } \\
\text { (min.) }\end{array}$ & $\begin{array}{c}\text { Lipo Cap. } \\
\text { Left (\%) }\end{array}$ & $\begin{array}{c}\text { Capt. } \\
\text { Probes }\end{array}$ \\
\hline PTSP & 2 & 7 & 85 & 14 & 33 & 54 \\
\hline CLSP & 2 & 7 & 85 & 17 & 25 & 34 \\
\hline SSP & 2 & 7 & 85 & 12 & 40 & 21 \\
\hline
\end{tabular}

TABLE VI. Results with a remote-controlled UAV over different SAR flight patterns.

A visual spotter was used to keep an eye on the ground control station to alert the pilot if altitude is exceeded beyond 85 feet to avoid the low quality of sensory data. Piloting the UAV to maintain a specific trajectory, e.g., in any of the three SAR flight patterns, can be challenging if the pilot does not have the necessary skills to maintain a precise flight pattern and if the sensor used requires a specific radius for each of the turns.

For this study, the Tetra sensor range can cover the turning areas and detect probes, even if the UAV misses on target the precise location of each waypoint rotation. This is one reason why the $\mathrm{RC}$ mode consumes more power from the LiPo but provides flexibility to the flight plan. An example is to slowly descend in a section of the search area having no trees to increase the probability of success to detect more probes.

During SAR operations, decisions need to be made to determine what search flight plan to follow, and the flight mode to execute based on the available resources. The autonomous flight mode leads to a low variability in the flight mission and more efficient power consumption. The RC flight mode requires skillful pilots to follow any of the three SAR flight patterns, consumes more power from the LiPo, provides flexibility during flight path, and captures more probes. The results yield a tradeoff between having a higher probability rate to detect probes with possibly longer flights, requiring more than one LiPo or executing the mission faster with one LiPo while detecting fewer probes. This is a decision any SAR teams must consider before executing the flight plan. 


\section{CHAPTER V}

\section{Concluding Remarks}

In this thesis, WiFi probe requests are captured from various cell phones using a Wi-Fi Pineapple mounted onto the UAV. Subsequently, using the information extracted from the probe requests captured, the RFA machine learning technique is used to find a coarse estimate (a predefined zone) for the Wi-Fi device's whereabouts. The experimental results demonstrate an accuracy of a $81.8 \%$ for finding the true zone location of a Wi-Fi device. The proposed approach is suited for search and rescue operations where a given area (e.g., different zones in a national forest) can be initially trained using the RFA method. Then, if a victim needs to be localized, the proposed method can be applied to find the zone where he/she is located. Our future work includes testing and training larger sets of data to evaluate the scalability of our proposed approach in larger geographical areas.

Then, the use of UAVs in SAR operations is presented to show its reliable support services. Vital issues to address and to understand before a UAV is deployed to conduct search and rescue missions are crucial to achieving a high probability of a successful SAR operation. Some other issues are aspects that affect the navigational performance of a UAV, how to bypass some of the flight time limitations of a UAV, and how the sensory operations are affected by the search flight patterns flown by the UAV. The results demonstrate the importance of correctly configuring the UAV to obtain the best possible flight performance and the longest flight time. Furthermore, knowing the flight plan to execute, the UAV can detect more Wi-Fi probe requests, which are crucial to respond quickly and increase the probability of having more accurate data to locate the victims. 
[1] X. Ma, Q.-Y. Huang, and X.-M. Shu, "A New Localization Algorithm of Mobile Phone for Outdoor Emergency Rescue," in Proc. IEEE Int. Conf. Intelligent Syst. Des. Eng. Appl. (ISDEA), Jan. 2013, pp. 124-127.

[2] Q. Hamp, R. Zhang, L. Chen, O. Gorgis, T. Ostertag, M. Loschonsky, and L. Reindl, "New technologies for the search of trapped victims," Ad Hoc Networks, vol. 13, pp. 69-82, 2014.

[3] A. Merwaday and I. Guvenc, "UAV assisted heterogeneous networks for public safety communications," in Proc. IEEE Wireless Commun. Netw. Conf. Workshops (WCNCW), Mar. 2015, pp. 329-334.

[4] E. K. Hung and D. A. Townes, "Search and rescue in Yosemite National Park: a 10-year review," Wilderness \& environmental medicine, vol. 18, no. 2, pp. 111-116, 2007.

[5] J. R. Kiran, "Design and implementation of mobile aerial nodes," Ph.D. dissertation, Birla Inst. Technol. and Science, Pilani, 2013.

[6] A. Kumbhar, F. Koohifar, I. Guvenc, and B. Mueller, "A Survey on Legacy and Emerging Technologies for Public Safety Communications," IEEE Commun. Surveys Tuts., 2016.

[7] A. Birk, B. Wiggerich, H. B. Low, M. Pfingsthorn, and S. Schwertfeger, "Safety, Security, and Rescue Missions with an Unmanned Aerial Vehicle (UAV)," Journal of Intelligent \& Robotic Systems, vol. 64, no. 1, pp. 57-76, 2011.

[8] E. Vattapparamban, I. Guvenc, A. Yurekli, K. Akkaya, and S. Uluagac, “,” in Proc. IEEE Int. Wireless Commun. Mobile Comp. Conf. (IWCMC), Paphos, Cyprus, Sep. 2016.

[9] S. Waharte and N. Trigoni, "Supporting search and rescue operations with UAVs," in Proc. IEEE Int. Conf. Emerging Security Tech.(EST), 2010, pp. 142-147.

[10] P. Rudol and P. Doherty, "Human body detection and geolocalization for UAV search and rescue missions using color and thermal imagery," in Proc. IEEE Aerospace Conf., Mar. 2008, pp. $1-8$.

[11] P. Doherty and P. Rudol, "A UAV search and rescue scenario with human body detection and geolocalization," in Australasian Joint Conf. Artificial Intelligence, Dec. 2007, pp. 1-13.

[12] M. Carpin, S. Rosati, M. E. Khan, and B. Rimoldi, "UAVs using Bayesian Optimization to Locate WiFi Devices," Preprint arXiv:1510.03592, 2015.

[13] J. Sundqvist, J. Ekskog, B. J. Dil, F. Gustafsson, J. Tordenlid, and M. Petterstedt, "Feasibility study on smartphone localization using mobile anchors in search and rescue operations," in Proc. IEEE Int. Conf. Inform. Fusion (FUSION), Jul. 2016, pp. 1448-1453.

[14] T. Hossmann, D. Schatzmann, P. Carta, and F. Legendre, "Twitter in disaster mode: smart probing for opportunistic peers," in Proc. ACM Int. Workshop Mobile Opportunistic Netw., 2012, pp. 93-94.

[15] Z. Liu, Y. Chen, B. Liu, C. Cao, and X. Fu, "HAWK: an unmanned mini-helicopter-based aerial wireless kit for localization," IEEE Trans. Mobile Computing, vol. 13, no. 2, pp. 287-298, Feb. 2014.

[16] H. Menouar, I. Guvenc, K. Akkaya, A. S. Uluagac, A. Kadri, and A. Tuncer, "UAV-Enabled Intelligent Transportation Systems for the Smart City: Applications and Challenges," IEEE Commun. Mag., 2016.

[17] T. Tomic, K. Schmid, P. Lutz, A. Domel, M. Kassecker, E. Mair, I. L. Grixa, F. Ruess, M. Suppa, and D. Burschka, "Toward a fully autonomous UAV: Research platform for indoor and outdoor urban search and rescue," IEEE Robot. Autom. Mag., vol. 19, no. 3, pp. 46-56, Sept. 2012. [18] Z. Xu, K. Sandrasegaran, X. Kong, X. Zhu, J. Zhao, B. Hu, and C.-C. Lin, "Pedestrian monitoring system using Wi-Fi technology and RSSI based localization," Int. J. Wireless \& Mobile Netw., vol. 5, no. 4, pp. 17-34, Aug. 2013. 
[19] E. Vattapparamban, B. S. C, iftler, 'I. Guvenc, K. Akkaya, and A. Kadri, "“ "Indoor occupancy tracking in smart buildings using passive sniffing of probe requests," in Proc. IEEE Int. Conf. Commun. Workshops (ICC), May 2016, pp. 38-44.

[20] A. B. M. Musa and J. Eriksson, "Tracking Unmodified Smartphones Using Wi-Fi Monitors," in Proc. ACM Conf. Embedded Netw. Sensor Syst., New York, NY, 2012, pp. 281-294.

[21] Wi-fi pineapple by hak5. [Online]. Available: https://www.wifipineapple. com/

[22] Busybox. [Online]. Available: http://www.busybox.net/

[23] Hak5. (2013) Wi-Fi Pineapple Mark V. [Online]. Available: http: //hakshop.myshopify.com/products/wifi-pineapple

[24] Mission Planner. [Online]. Available: http://planner.ardupilot.com/

[25] L. Breiman, "Random forests," Machine Learning, vol. 45, pp. 5-32, 2001. [26] "Bagging Predictors," Machine Learning, vol. 24 no. 2, pp. 123- 140, 1996.

[26] _ _ "Bagging Predictors," Machine Learning, vol. 24 no. 2, pp. 123-140, 1996.

[27] A. Matos, E. Silva, N. Cruz, J. C. Alves, D. Almeida, M. Pinto, A. Martins, J. Almeida, and D. Machado, "Development of an unmanned capsule for large-scale maritime search and rescue," in Proceedings IEEE OCEANS, Sept. 2013, pp. 1-8.

[28] S. Waharte and N. Trigoni, "Supporting search and rescue operations with UAVs," in Proceedings Emerging Security Technologies (EST), Sept. 2010.

[29] G. De Cubber, D. Daniela, S. Daniel, C. Keshav, S. Rui, and O. Stephane, "The eu-icarus project: Developing assistive robotic tools for search and rescue operations," in Proc. 2013 IEEE International Symposium on Safety, Security, and Rescue Robotics (SSRR), Oct. 2013.

[30] Turic, Dujmic, and Papic, "Two-stage segmentation of aerial images for search and rescue," Information Technology and Control, vol. 39, pp. 138-145, 2010.

[31] R. Bloss, "Unmanned Vehicles while becoming smaller and smarter are addressing new applications in medical, agriculture, in addition to military and security," Industrial Robot: An International Journal, vol. 41, pp. 82-86, 2014.

[32] P. Jesper, "Use of UAVs in the NGO world," in CRS Conference ICT4 Development, March. 2014.

[33] G. Lewis and J. Li, "Evaluating the use of a low-cost Unmanned Aerial Vehicle platform in acquiring digital imagery for emergency response," Geomatics solutions for disaster management, pp. 117-133, 2007.

[34] S. M. Adams and F. C.J., "A survey of Unmanned Aerial Vehicle (UAV) usage for imagery collection in disaster research and management," in 9th Int. Workshop on Remote Sensing for Disaster Response, 2011.

[35] T. Smedley. (2015) Drones new mission: saving lives in developing countries. [Online]. Available: https:/www.theguardian.com/sustainable-business/2015/jan/09/ drones-tech-naturaldisasters-medical-developing-countries

[36] R. Hlund. (2017) Search and Rescue (SAR) using UAVs. [Online]. Available: http://smartplanes.se/search-and-rescue-sar-using-uavs/

[37] M. Quaritsch, R. Kuschnig, H. Hellwagner, and B. Rinner, "Fast aerial image acquisition and mosaicking for emergency response operations by collaborative UAVs," in Int. Conf. on Inform. Syst. for Crisis Response and Manag., May 2011, p. 5.

[38] S. Verykokou, A. Doulamis, and G. Athanasiou, "UAV-based 3d modeling of disaster scenes for urban search and rescue," in Proc. IEEE Int. Conf. Imaging Systems and Techniques (IST), Nov. 2016. 
[39] Pix4D. (2017) Drone mapping supports search \& rescue operations during Colombia landslide. [Online]. Available: http:/www.unmannedsystemstechnology.com/2017/05/ dronemapping-supports-search-rescue-operations-colombia-landslide/

[40] A. Ferworn Ryerson, S. Herman, J. Tran, A. Ufkes, and R. Mcdonald, "Disaster scene reconstruction: modeling and simulating urban building collapse rubble within a game engine," in Proc. Summer Computer Simulation, July 2013.

[41] J. Sokalski, T. P. Breckon, and I. Cowling, "Automatic salient object detection in UAV imagery," in Proc. Int. Conf. in UAV Systems, April 2010, pp. 11.1-11.12.

[42] M. Goodrich, J. Cooper, J. Adams, C. Humphrey, R. Zeeman, and B. Buss, "Using a miniUAV to support wilderness search and rescue: Practices for human-robot teaming," in Proc. IEEE Int. Workshop on Safety, Security and Rescue Robotics, Sept. 2007, pp. 11.1-11.12.

[43] S. Jingxuan, L. Boyang, J. Yifan, and W. Chih-Yung, "A camera-based target detection and positioning UAV system for search and rescue (SAR) purposes," Sensors, vol. 16, no. 11, pp. 124, 2016.

[44] T. Tomic, K. Schmid, P. Lutz, A. Domel, M. Kassecker, E. Mair, I. Grixa, F. Ruess, M. Suppa, and D. Burschka, "Toward a fully autonomous UAV: Research platform for indoor and outdoor urban search and rescue," IEEE Robot, vol. 19, pp. 46-56, 2012.

[45] T. Chien-Ming, C. Chi-Kin, E. Khaled, and K. Majid. (2017) Flight tour planning with recharging optimization for battery-operated autonomous drones. [Online]. Available: http://arxiv.org/abs/1703.10049

[46] B. Saha, C. Quach, and K. Goebel, "Optimizing battery life for electric UAVs using a Bayesian framework," in Proc. IEEE Aerospace Conference, Mar. 2012, pp. 11.1-11.12.

[47] D. Namiot and M. Sneps-Sneppe, "On the analysis of statistics of mobile visitors," Automatic Control and Computer Sciences, vol. 48, pp. 150- 158, 2014.

[48] A. Musa and J. Eriksson, "Tracking unmodified smartphones using WiFi monitors," in Proc. ACM Conf. on Embedded Networked Sensor Systems (SenSys), Nov. 2012, pp. 281-294.

[49] A. Di Luzio, A. Mei, and J. Stefa, "Mind Your Probes: DeAnonymization of Large Crowds Through Smartphone WiFi Probe Requests," in Proc. IEEE INFOCOM , 2016.

[50] Hak5. (2015) Wi-Fi Pineapple Tetra. [Online]. Available: https: //wifipineapple.com/pages/tetra

[51] W. Wang, R. Joshi, A. Kulkarni, W. Kay Leong, and B. Leong, "Feasibility study of mobile phone WiFi detection in aerial search and rescue operations," in Proc. ACM Asia-Pacific Workshop on Systems, July 2013.

[52] Wireshark. [Online]. Available: https://www.wireshark.org/

[53] Work smarter with drone date. [Online]. Available: https://www. dronedeploy.com/

[54] FAA. (2017) Fly for Fun under the Special Rule for Model Aircraft. [Online]. Available: https://www.faa.gov/uas/getting started/fly for fun/

[55] S. Benkoski, M. Monticino, and J. Weisinger, "A survey of the search theory literature," Naval Research Logistics, vol. 38, pp. 469-494, 1991.

[56] H. Loewen. (2013) Isolating components from UAV vibration. [Online]. Available: https://www.micropilot.com/pdf/ isolating-components-uav-vibration.pdf

[57] Batteries for UAVs. [Online]. Available: http://dronesarefun.com/BatteriesForUAV html.

[58] LearningRC. (2017) RC LIPO BATTERY GUIDE: EXPLANATION, SAFETY, AND CARE. [Online]. Available: http://learningrc.com/ lipo-battery/

[59] O. Liang. (2017) HOW TO CHOOSE BATTERY CAPACITY FOR LONGER FLIGHT TIME. [Online]. Available: https://oscarliang.com/ how-to-choose-battery-for-quadcoptermulticopter/ 
[60] IMO/ICAO, International Aeronautical and Maritime Search and Rescue Manual. IAMSR, 2010.

[61] V. Acuna, K. Kumbhar, E. Vattapparamban, F. Rajabli, and I. Guvenc, "Localization of WiFi Devices Using Probe Requests Captured at Unmanned Aerial Vehicles," in Proc. IEEE Wireless Communications and Networking Conf (WCNC), 2017. 